University of Nebraska - Lincoln DigitalCommons@University of Nebraska - Lincoln

1990

\title{
High- Resolution Shock-Capturing Schemes for Inviscid and Viscous Hypersonic Flows
}

Helen C. Yee

NASA Ames Research Center, yee@nas.nasa.gov

G. H. Klopfer

NEAR Inc.

J.-L. Montagne

ONERA

Follow this and additional works at: http://digitalcommons.unl.edu/nasapub

Yee, Helen C.; Klopfer, G. H.; and Montagne, J.-L., "High- Resolution Shock-Capturing Schemes for Inviscid and Viscous Hypersonic Flows" (1990). NASA Publications. 281.

http://digitalcommons.unl.edu/nasapub/281

This Article is brought to you for free and open access by the National Aeronautics and Space Administration at DigitalCommons@University of Nebraska - Lincoln. It has been accepted for inclusion in NASA Publications by an authorized administrator of DigitalCommons@University of Nebraska - Lincoln. 


\title{
High-Resolution Shock-Capturing Schemes for Inviscid and Viscous Hypersonic Flows*
}

\author{
H. C. YeE \\ NASA Ames Research Center, \\ Moffett Field, California 94035 \\ G. H. KLOPFER \\ NEAR Inc., Mountain View, California 94043
}

AND

J.-L. Montagné ${ }^{\dagger}$

ONERA, B.P. 72, 92322 Chatillon Cedex, France

Received April 28, 1988; revised January 13, 1989

\begin{abstract}
A class of high-resolution implicit total variation diminishing (TVD) type algorithms suitable for transonic multidimensional Euler and Navier-Stokes equations has been extended to hypersonic computations. The improved conservative shock-capturing schemes are spatially second- and third-order and are fully implicit. They can be first- or second-order accurate in time and are suitable for either steady or unsteady calculations. Enhancement of stability and convergence rate for hypersonic flows is discussed. With the proper choice of the temporal discretization and implicit linearization, these schemes are fairly efficient and accurate for very complex two-dimensional hypersonic inviscid and viscous shock interactions. This study is complemented by a variety of steady and unsteady viscous and inviscid hypersonic bluntbody flow computations. Due to the inherent stiffness of viscous flow problems, numerical experiments indicated that the convergence rate is in general slower for viscous flows than for inviscid steady flows. (C) 1990 Academic Press, Inc.
\end{abstract}

\section{Motivation AND OBJective}

Most shock-capturing methods are either inefficient for practical computations or only valid for transonic or supersonic perfect gas calculations. For hypersonic,

* An abbreviated version appeared in the proceedings of the BAIL V Conference, June 20-24, 1988, Shanghai, China. The full text was published as a preliminary report-NASA Technical Memorandum 100097, April 1988.

${ }^{\dagger}$ This work was performed while on leave as an Ames Associate at NASA Ames Research Center, Moffett Field, CA 94035. 
perfect gas, equilibrium real gases, or nonequilibrium flows, improvement and modification to existing methods are necessary. In addition, viscous hypersonic and nonequilibrium flow problems are generally stiff and implicit methods are often preferred over explicit methods. Some of the numerical issues for steady inviscid hypersonic blunt-body flow computations were addressed in our earlier paper [1]. A semi-implicit method and a fully implicit method for steady-state nonequilibrium flows were discussed in Yee and Shinn [2]. A basic study on numerical methods for unsteady inviscid nonequilibrium flows was presented in LeVeque and Yee [3]. The objective of this research is to efficiently extend and improve, as well as to present, a unified formulation of the existing implicit high-resolution shock-capturing schemes $[4-6,2]$ for multidimensional compressible Euler and Navier-Stokes equations in the hypersonic, perfect, and equilibrium real gas flow regimes.

The improved schemes are based on a class of implicit total variation diminishing (TVD) type algorithms originally designed for transonic and supersonic multidimensional Euler and Navier-Stokes equations [4-6]. The extended conservative shock-capturing schemes are spatially second- and third-order and are fully implicit. They can be first- or second-order accurate in time and are suitable for either steady or unsteady calculations. Here, the time and spatial order of accuracy pertains to the scheme for nonlinear scalar hyperbolic conservation laws with uniform grid spacing, and the spatial accuracy is for numerical solutions that are away from discontinuities. In addition, the current unified formulation allows the inclusion of the MUSCL-type approach [7] in conjunction with a local characteristic approach $[24,6]$ or flux-vector splittings [8] (see Section II for an explanation). For the present study, particular emphasis is placed on second-order implicit time-accurate high-resolution algorithms. The algorithms are formulated in finite volume and pseudo finite volume forms which, for certain physical problems and grid distributions, can enhance stability and convergence rate for highly clustered or skewed grids and require only a slight modification from the form originally presented in Yee and Harten [5] for generalized geometries. It is emphasized here that the use of the term TVD-type schemes pertains to the property of the algorithm as applied to one-dimensional nonlinear scalar hyperbolic conservation laws or constant coefficient hyperbolic systems, and the TVD property is in a semidiscrete sense for the second-order time discretization. Theoretical justification of the proposed fully discretized schemes on the preservation of TVD property for the general nonlinear scalar hyperbolic conservation laws is an open question and requires further investigation. Moreover, the high-resolution property of these schemes for multidimensional nonlinear systems of hyperbolic conservation laws is evaluated by numerical experiments. In particular, the following numerical issues are addressed:

1. Some numerical aspects of TVD-type schemes that affect the convergence rate for hypersonic Mach numbers and real gas flows but have negligible effect on low Mach number or perfect gas flows are identified.

2. The performance of the various linearized implicit forms of the proposed 
schemes similar to the transonic flow study [4-6] is reexamined for hypersonic flows.

3. The behavior of the proposed schemes with various temporal differencing but similar spatial discretization for inviscid and viscous flows is investigated. Studies indicated that their behavior in terms of stability and convergence rate is quite different between viscous and inviscid flows. However, with the proper choice of the temporal discretization and suitable implicit linearization, these schemes are fairly efficient and accurate for very complex two-dimensional hypersonic inviscid and viscous shock interactions.

4. The relative efficiency and accuracy of typical TVD-type schemes [9-11] for shock wave computations is examined. A comparative study on steady and unsteady flows reveals that the proposed class of TVD-type schemes, in particular, for equilibrium real gas and nonequilibrium flows, produces just as accurate shock resolution and yet requires less operations count than most other TVD schemes (e.g., higher order Godunov [10], Osher and Chakravarthy [9], and TVD fluxvector splitting approaches [8]).

Numerical experiments from four of our conference papers $[1,11,21,22]$ have been integrated into the behavior and performance of our schemes concluded in this paper. Readers are encouraged to refer to these references for more details. Most of the numerical solutions are compared with the exact solutions or experimental data, or compared with existing schemes. In the following section, the generalization of Roe's approximate Riemann solver and flux-vector splitting for real gases is reviewed. A description of the two-parameter family implicit shock-capturing scheme, the various enhancements on numerical stability and convergence rate for hypersonic flows, and the behavior of the scheme for inviscid and viscous flows are discussed in the subsequent sections. To illustrate the performance of the schemes for complex hypersonic flows, some representative numerical examples are also discussed.

\section{Description of the Numerical Algorithm}

The conservation laws for the two-dimensional Navier-Stokes equations can be written in the form

$$
\frac{\partial U}{\partial t}+\frac{\partial F}{\partial x}+\frac{\partial G}{\partial y}=\frac{1}{\operatorname{Re}}\left[\frac{\partial F_{v}}{\partial x}+\frac{\partial G_{v}}{\partial y}\right]
$$

where $\quad U=[\rho, m, n, e]^{\mathrm{T}}, \quad F=[\rho u, m u+p, n u, e u+p u]^{\mathrm{T}}, \quad G=[\rho v, m v, n v+p$, $e v+p v]^{\mathrm{T}}, F_{v}=\left[0, \tau_{x x}, \tau_{x y}, f\right]^{\mathrm{T}}$, and $G_{v}=\left[0, \tau_{x y}, \tau_{y y}, g\right]^{\mathrm{T}}$. Here $\rho$ is the density, $u$ and $v$ are the velocity components, $m=\rho u$ and $n=\rho v$ are the $x$ - and $y$-components of the momentum per unit volume, $p$ is the pressure, $\varepsilon=\rho\left[\varepsilon+\frac{1}{2}\left(u^{2}+v^{2}\right)\right]$ is the 
total energy per unit volume, and $\varepsilon$ is the specific internal energy. For a perfect gas, we also have

$$
\begin{aligned}
\tau_{x x} & =\mu\left(4 u_{x}-2 v_{y}\right) / 3 \\
\tau_{x y} & =\mu\left(u_{y}+v_{x}\right) \\
\tau_{y y} & =\mu\left(-2 u_{x}+4 v_{y}\right) / 3 \\
f & =u \tau_{x x}+v \tau_{x y}+\mu \operatorname{Pr}^{-1}(\gamma-1)^{-1} \frac{\partial a^{2}}{\partial x} \\
g & =u \tau_{x y}+v \tau_{y y}+\mu \operatorname{Pr}^{-1}(\gamma-1)^{-1} \frac{\partial a^{2}}{\partial y}
\end{aligned}
$$

where, for example, $u_{x}$ is defined as $\partial u / \partial x$. The dynamic viscosity $\mu$ is given by Sutherland's formula. The Reynolds number is Re, the Prandtl number is Pr, the sound speed is $a$, and the ratio of specific heats is $\gamma$.

Under a generalized coordinate transformation, $\xi=\xi(x, y)$ and $\eta=\eta(x, y)$, Eqs. (1) can be written in a form which maintains the strong conservation-law form as

$$
\frac{\partial \hat{U}}{\partial t}+\frac{\partial \hat{F}}{\partial \xi}+\frac{\partial \hat{G}}{\partial \eta}=\frac{1}{\operatorname{Re}}\left[\frac{\partial \hat{F}_{v}}{\partial \xi}+\frac{\partial \hat{G}_{v}}{\partial \eta}\right],
$$

where $\hat{U}=U / J, \hat{F}=\left(\xi_{x} F+\xi_{y} G\right) / J, \hat{G}=\left(\eta_{x} F+\eta_{y} G\right) / J, \hat{F}_{v}=\left(\xi_{x} F_{v}+\xi_{y} G_{v}\right) / J, \hat{G}_{v}=$ $\left(\eta_{x} F_{v}+\eta_{y} G_{v}\right) / J$, and $J=\xi_{x} \eta_{y}-\xi_{y} \eta_{x}$, the Jacobian transformation. Let $A=\partial F / \partial U$ and $B=\partial G / \partial U$. Then the Jacobians $\hat{A}=\partial \hat{F} / \partial \hat{U}$ and $\hat{B}=\partial \hat{G} / \partial \hat{U}$ can be written as

$$
\begin{aligned}
& \hat{A}=\left(\xi_{x} A+\xi_{y} B\right) \\
& \hat{B}=\left(\eta_{x} A+\eta_{y} B\right) .
\end{aligned}
$$

In this study the thin-layer Navier-Stokes approximation is assumed by dropping all the $\partial(\cdot) / \partial \xi$ derivatives in the viscous terms. Also, stability and convergence rate viscous results are for a perfect gas and laminar flows with adiabatic wall conditions.

\subsection{Riemann Solvers}

Here the usual approach of applying the one-dimensional scalar TVD schemes via the so-called Riemann solvers for each direction in multidimensional nonlinear systems of hyperbolic conservation laws (see, for example, Refs. [5, 12]) are used. This approach is best suited for orthogonal or nearly orthogonal grids. The eigenvalues and eigenvectors of the Jacobian matrices $\hat{A}$ and $\hat{B}$ are used in approximate Riemann solvers. Given two states whose difference is $\Delta U$, Roe [13] obtained an average $\bar{A}$ in the $\xi$-direction, for example, satisfying $\Delta \hat{F}=\bar{A} \Delta U$ for a perfect gas. The generalization by Vinokur [14] for an arbitrary gas involves the pressure 
derivatives $\chi=(\partial p / \partial \rho)_{\tilde{\varepsilon}}$ and $\kappa=(\partial p / \partial \tilde{\varepsilon})_{\rho}$, where $\tilde{\varepsilon}=\rho \varepsilon$. The relation $c^{2}=\chi+\kappa h$ then gives the speed of sound, where $h=\varepsilon+p / \rho$. Introducing $H=h+\left(u^{2}+v^{2}\right) / 2$, Vinokur found the same expressions for $\bar{u}, \bar{v}$, and $\bar{H}$ for the perfect gas, and that $\bar{\chi}$ and $\bar{\kappa}$ must satisfy

$$
\bar{\chi} \Delta \rho+\bar{\kappa} \Delta \tilde{\varepsilon}=\Delta p .
$$

Unique values of $\bar{\chi}$ and $\bar{\kappa}$ are obtained by projecting the proper averages of the values for the two states into this relation (see Refs. $[14,11,12,15]$ for the exact formulas).

Flux-vector splitting methods divide the flux $\hat{F}$ into several parts, each of which has a Jacobian matrix whose eigenvalues are all of one sign. The approach by Steger and Warming [16] made use of the relation $F=A U$, valid for a perfect gas. Van Leer [17] constructed a different splitting in which the eigenvalues of the split-flux Jacobians are continuous and one of them vanishes, leading to sharper capture of transonic shocks. Vinokur and Montagné [18] showed that the expressions for both these splittings can be generalized to an arbitrary gas by using the variable $\gamma=\rho c^{2} / p$, and adding to the split energy flux a term equal to the product of the split mass flux and the quantity $\varepsilon-c^{2} /[\gamma(\gamma-1)]$ (see Refs. $[18,11,12,15]$ for the exact formulas).

The current study on the shock resolution of the various schemes $[1,4-6,9]$ for two-dimensional steady-state blunt-body inviscid computations indicates similar trends as the one-dimensional study [11]. These schemes produce similar shockresolution, but the main issue appears to be their relative efficiency. Due to extra evaluations per dimension in the curve fitting between the left and right states in a real gas for the van Leer formulation, additional computation is required for the van Leer type schemes than the Harten and Yee $[4,5,19]$ and Yee [6] types of TVD schemes. Here van Leer type schemes refer to the use of the MUSCL approach [7] (see Section 2.2 for an explanation) in conjunction with the Roe type approximate Riemann solver [13] or flux-vector splittings [8] (hereafter the latter methods are referred to as the TVD flux-vector splitting methods). Moreover, for steady-state applications, implicit methods are preferred over explicit methods because of the faster convergence rate (in terms of CPU-time). In addition, it is easier to obtain a noniterative linearized implicit operator for the Harten and Yee, and Yee type schemes than for the van Leer type schemes. Furthermore, unlike flux-vector splitting approaches, implicit methods employing the Roe type approximate Riemann solver (non-MUSCL or MUSCL) with first-order implicit operators do not require the Jacobian of the $F^{ \pm}$and $G^{ \pm}$fluxes. Here $F^{ \pm}$is the portion of the flux with positive/negative eigenvalues. In many instances, the Jacobians of these fluxes are relatively difficult or expensive to obtain, in particular, for nonequilibrium flows. A similar difficulty applies to the MUSCL formulations via the Roe type approximate Riemann solver if a spatially second-order implicit operator is desired. For these reasons, the linearized implicit versions of Harten and Yee [4] and Yee [6] are preferred over the van Leer type schemes. Consequently, 
numerical studies on the extension of the former schemes to hypersonic flows are emphasized. Some of these points will become more apparent when an unified formulation of these implicit methods is presented in Section 2.2. An unified formulation of the corresponding explicit schemes can be found in Ref. [12].

\subsection{Description of the Implicit TVD Schemes}

In the application of TVD-type schemes for viscous flows, the physical problems considered here are assumed to be inviscid dominated, in the sense that moderate or strong shock waves are present in the flow field such that high-resolution shockcapturing techniques are required. Thus the numerical procedures used here for the compressible Navier-Stokes calculations are a second-order central difference approximation for the diffusion terms and TVD-type schemes for the inviscid part of the Navier-Stokes equations. The question of whether the present numerical dissipation term (due to the TVD-type terms) has an adverse effect on the true viscosity terms in the boundary layer region is not known at this point. What we can conclude from the current study is that the portions of the solution slightly or far away from the boundary layer are quite accurately simulated.

The two-parameter family of explicit and implicit high-resolution schemes presented here is based on a semi-discrete methodology and on the one-parameter family of TVD-type algorithms developed in Refs. $[19,4-6]$. The idea is to use the same spatial discretization as references $[19,4-6]$ for the spatial derivatives and to use the two-parameter family of linear multistep methods for the time derivatives. The original one-parameter family of TVD-type schemes is a subset of the twoparameter family of algorithms. Mathematical analysis similar to that in $[19,4-6]$ for the current larger family of schemes requires further investigation. For a particular chosen time differencing, these schemes are TVD for the one-dimensional constant coefficient hyperbolic equations. Also the MUSCL approach in conjunction with the Roe-type approximate Riemann solver [2] and TVD flux-vector splitting methods [8] falls nicely into the present framework. In other words, the present formulation includes a larger class of spatial as well as temporal discretization than in Refs. $[19,4-6]$.

The formulation is in finite volume and pseudo finite volume forms which can enhance stability and convergence rate (in terms of allowing a larger time step and requiring fewer iterations) for highly clustered or skewed grids and is a slight modification of the form originally presented in Yee and Harten [5] for generalized geometries. For fairly uniform or mildly clustered grids, the present finite volume and pseudo finite volume forms behave the same as in Ref. [5] for inviscid flows. This is in contrast to the study of Takakura et al. [20] which claimed that their modified form [20] is the correct finite difference formulation for generalized geometries. A comparison between Takakura et al. [20] and Ref. [5] on the same fairly uniform curvilinear grid for a blunt-body computation shows no noticeable difference in resolution.

Without loss of generality, the two-parameter family of implicit schemes for the Euler equations $\left(F_{v}=G_{v}=0\right)$ is presented here. For general Navier-Stokes equa- 
tions, the appropriate three-point central differences of the viscous Jacobian terms should be added to the implicit operator and a central difference approximation for the diffusion terms should be added to the explicit operator. For time-accurate Navier-Stokes calculations or for faster convergence to steady state, the viscous Jacobian terms were found to be necessary.

Let $\Delta t$ be the time step and let the grid spacing be denoted by $\Delta \xi$ and $\Delta \eta$ such that $\xi=j \Delta \xi$ and $\eta=k \Delta \eta$. Also let $\lambda^{\xi}=\Delta t / \Delta \xi$ and $\lambda^{\eta}=\Delta t / \Delta \eta$; then a twoparameter family of explicit and implicit TVD-type algorithms in generalized coordinates for two-dimensional systems (1) with $F_{v}=G_{v}=0$ can be written as

$$
\begin{aligned}
\Delta \hat{U}_{j, k}^{n}+ & \frac{\lambda^{\xi} \theta}{1+\omega}\left[\tilde{F}_{j+1 / 2, k}^{n+1}-\tilde{F}_{j-1 / 2, k}^{n+1}\right]+\frac{\lambda^{n} \theta}{1+\omega}\left[\tilde{G}_{j, k+1 / 2}^{n+1}-\widetilde{G}_{j, k-1 / 2}^{n+1}\right] \\
= & -\frac{(1-\theta) \lambda^{\xi}}{1+\omega}\left[\tilde{F}_{j+1 / 2, k}^{n}-\tilde{F}_{j-1 / 2, k}^{n}\right]-\frac{(1-\theta) \lambda^{n}}{1+\omega}\left[\tilde{G}_{j, k+1 / 2}^{n}-\tilde{G}_{j, k-1 / 2}^{n}\right] \\
& +\frac{\omega}{1+\omega} \Delta \hat{U}_{j, k}^{n-1} .
\end{aligned}
$$

Here $\Delta \hat{U}_{j, k}^{n}=\hat{U}_{j, k}^{n+1}-\hat{U}_{j, k}^{n}$. The functions $\widetilde{F}_{j+1 / 2, k}$ and $\widetilde{G}_{j, k+1 / 2}$ are the numerical fluxes in the $\xi$ - and $\eta$-directions evaluated at $\left(j+\frac{1}{2}, k\right)$ and $\left(j, k+\frac{1}{2}\right)$, respectively. This two-parameter family of algorithms contains first- and second-order implicit as well as explicit schemes. The scheme is temporally second order if $\theta=\omega+\frac{1}{2}$ and first order otherwise. When $\theta \neq 0$, algorithm (5) is an implicit scheme. In this paper, only the temporally first-order backward Euler $(\theta=1, \omega=0)$ and the temporally second-order three-point backward differentiation $\left(\theta=1, \omega=\frac{1}{2}\right)$ time-differencing are investigated. Detailed formulation and numerical studies for algorithm (5) with $\omega=0$ for transonic, supersonic, and hypersonic flows can be found in references $[4-6,12,1,11,21,22]$. The current study shows that, for viscous steady and unsteady flows, the temporally second-order implicit algorithm $\left(\theta=1, \omega=\frac{1}{2}\right)$ appears to be slightly more stable and efficient than the temporally first-order implicit algorithm $(\theta=1, \omega=0)$.

The spatial accuracy of Eq. (5) depends on the form of the numerical flux functions. There exist many ways to achieve higher order spatial accuracy and at the same time have TVD-type properties. Here two of the ways are discussed. The first is due to Harten [19], Roe [23], and Yee, Roe, and Davis [6, 24, 25], and the second, sometimes referred to as the MUSCL approach, is due to van Leer [7]. Following the same nomenclature as in Refs. [11,12], hereafter, we refer to the first way as the non-MUSCL approach. Besides the different temporally implicit discretization, the combination of the two Riemann solvers discussed in Section 2.1 and higher order spatial differencing considered yields three different types of spatial differencing for the nonlinear system (1): namely, non-MUSCL and MUSCL approaches using an approximate Riemann solver, and a MUSCL approach using flux-vector splittings (TVD flux-vector splitting methods). 
Non-MUSCL approach using an approximate Riemann solver. The numerical flux function $\tilde{F}_{j+1 / 2, k}$ for a non-MUSCL type approach, together with the local characteristic approach [4-6] (Roe type of approximate Riemann solver) in a finite volume formulation, can be expressed as (omitting the time superscript)

$$
\begin{aligned}
\tilde{F}_{j+1 / 2, k}= & \frac{1}{2}\left[\left(\frac{\xi_{x}}{J}\right)_{j+1 / 2}\left(F_{j, k}+F_{j+1, k}\right)+\left(\frac{\xi_{y}}{J}\right)_{j+1 / 2}\right. \\
& \left.\times\left(G_{j, k}+G_{j+1, k}\right)+R_{j+1 / 2} \Phi_{j+1 / 2} / J_{j+1 / 2}\right] .
\end{aligned}
$$

The corresponding pseudo finite volume formulation will be discussed in Section 2.4. Here $F_{j, k}=F\left(\hat{U}_{j, k}\right)$ and $R_{j+1 / 2}$ is the matrix whose columns are the eigenvectors of $\partial \hat{F} / \partial U$ evaluated at some symmetric average of $U_{j, k}$ and $U_{j+1, k}$ (for example, Roe average [13] for a perfect gas and generalized Roe average of Vinokur [14] for real gases). The explicit form of $R_{j+1 / 2}$ can be found in Refs. $[38,13,14,37]$. To simplify the notation, the subscript $j+\frac{1}{2}$ is used to mean $j+\frac{1}{2}, k$. The values

$$
\begin{aligned}
\left(\frac{\xi_{x}}{J}\right)_{j+1 / 2} & =\frac{1}{2}\left[\left(\frac{\xi_{x}}{J}\right)_{j, k}+\left(\frac{\xi_{x}}{J}\right)_{j+1, k}\right] ; \\
\frac{1}{J_{j+1 / 2}} & =\frac{1}{2}\left(\frac{1}{J_{j+1, k}}+\frac{1}{J_{j, k}}\right) .
\end{aligned}
$$

Also

$$
\left(k_{1}\right)_{j+1 / 2}=\left[\frac{\xi_{x}}{J} / \sqrt{\left(\frac{\xi_{x}}{J}\right)^{2}+\left(\frac{\xi_{y}}{J}\right)^{2}}\right]_{j+1 / 2}
$$

and

$$
\left(k_{2}\right)_{j+1 / 2}=\left[\frac{\xi_{y}}{J} / \sqrt{\left(\frac{\xi_{x}}{J}\right)^{2}+\left(\frac{\xi_{y}}{J}\right)^{2}}\right]_{j+1 / 2}
$$

used in $R_{j+1 / 2}$ are defined, for example, as

$$
\left(k_{1}\right)_{j+1 / 2}=\left[\left(\frac{\xi_{x}}{J}\right)_{j+1 / 2} / \sqrt{\left(\frac{\xi_{x}}{J}\right)_{j+1 / 2}^{2}+\left(\frac{\xi_{y}}{J}\right)_{j+1 / 2}^{2}}\right] .
$$

The values $\xi_{x}, \xi_{y}, \eta_{x}$, and $\eta_{y}$ are evaluated by three-point central differences. Similarly, one can define the numerical flux $\widetilde{G}_{j, k+1 / 2}$ in this manner.

Here the form of $\Phi_{j+1 / 2}$ can be divided into two types: (a) a spatially secondorder symmetric TVD-type scheme $[6,24,25]$ in which the numerical dissipation terms are independent of the sign of the characteristic speeds and (b) a spatially second-order upwind TVD-type scheme $[19,5]$ in which the numerical dissipation terms depend on the sign of the characteristic speeds. 
The elements of the $\Phi_{j+1 / 2}$ in the $\xi$-direction denoted by $\left(\phi_{j+1 / 2}^{l}\right)^{S}$ for a spatially second-order symmetric TVD-type scheme $[6,12]$ are

$$
\left(\phi_{j+1 / 2}^{l}\right)^{S}=-\psi\left(a_{j+1 / 2}^{l}\right)\left[\alpha_{j+1 / 2}^{l}-\hat{Q}_{j+1 / 2}^{l}\right] .
$$

The value $a_{j+1 / 2}^{l}$ is the characteristic speed $a^{l}$ for $\partial \hat{F} / \partial \hat{U}$ evaluated at the same symmetric average between $U_{j, k}$ and $U_{j+1, k}$. The function $\psi$ is

$$
\psi(z)= \begin{cases}|z|, & |z| \geqslant \delta_{1} \\ \left(z^{2}+\delta_{1}^{2}\right) / 2 \delta_{1} & |z|<\delta_{1} .\end{cases}
$$

Here $\psi(z)$ in Eq. (7b) is an entropy correction to $|z|$, where $\delta_{1}$ is a small positive parameter $[12,33]$. For problems containing simple unsteady shocks, $\delta_{1}$ is set to zero in most of the computations since entropy-violating phenomena occur only for steady or nearly steady shocks. For steady-state problems containing strong shock waves, a proper control of the size of $\delta_{1}$ is very important, especially for hypersonic blunt-body flows. The choice of $\delta_{1}$ is also highly dependent on the Mach number and geometry of the physical problem. For the current numerical examples, the parameter $\delta_{1}$ is set to be a function of $a_{\xi}^{l}$ and $a_{\eta}^{l}$. See Ref. [12] or Section III for a discussion.

Examples of the "limiter" function $\hat{Q}_{j+1 / 2}^{l}$ can be expressed as

$$
\begin{aligned}
& \hat{Q}_{j+1 / 2}^{l}=\operatorname{minmod}\left(\alpha_{j-1 / 2}^{l}, \alpha_{j+1 / 2}^{l}\right)+\operatorname{minmod}\left(\alpha_{j+1 / 2}^{l}, \alpha_{j+3 / 2}^{l}\right)-\alpha_{j+1 / 2}^{l} \\
& \hat{Q}_{j+1 / 2}^{l}=\operatorname{minmod}\left(\alpha_{j-1 / 2}^{l}, \alpha_{j+1 / 2}^{l}, \alpha_{j+3 / 2}^{l}\right) \\
& \hat{Q}_{j+1 / 2}^{l}=\operatorname{minmod}\left[2 \alpha_{j-1 / 2}^{l}, 2 \alpha_{j+1 / 2}^{l}, 2 \alpha_{j+3 / 2}^{l}, \frac{1}{2}\left(\alpha_{j-1 / 2}^{l}+\alpha_{j+3 / 2}^{l}\right)\right] .
\end{aligned}
$$

The minmod function of a list of arguments is equal to the smallest number in absolute value if the list of arguments is of the same sign, or is equal to zero if any arguments are of opposite sign. Here $\alpha_{j+1 / 2}^{l}$ are elements of

$$
\alpha_{j+1 / 2}=R_{j+1 / 2}^{-1}\left(U_{j+1, k}-U_{j, k}\right) .
$$

The elements of the $\Phi_{j+1 / 2}$ in the $\xi$-direction denoted by $\left(\phi_{j+1 / 2}^{l}\right)^{U}$ for a spatially second-order upwind TVD-type scheme $[19,5,12]$ are

$$
\left(\phi_{j+1 / 2}^{l}\right)^{U}=\frac{1}{2} \psi\left(a_{j+1 / 2}^{l}\right)\left(g_{j+1}^{l}+g_{j}^{l}\right)-\psi\left(a_{j+1 / 2}^{l}+\gamma_{j+1 / 2}^{l}\right) \alpha_{j+1 / 2}^{l},
$$

where

$$
\gamma_{j+1 / 2}^{l}=\frac{1}{2} \psi\left(a_{j+1 / 2}^{l}\right) \begin{cases}\left(g_{j+1}^{l}-g_{j}^{l}\right) / \alpha_{j+1 / 2}^{l}, & \alpha_{j+1 / 2}^{l} \neq 0, \\ 0, & \alpha_{j+1 / 2}^{l}=0 .\end{cases}
$$

Examples of limiter function $g_{j}^{l}$ used in calculations are

$$
\begin{aligned}
& g_{j}^{l}=\operatorname{minmod}\left(\alpha_{j-1 / 2}^{l}, \alpha_{j+1 / 2}^{l}\right) \\
& g_{j}^{l}=\left(\alpha_{j+1 / 2}^{l} \alpha_{j-1 / 2}^{l}+\left|\alpha_{j+1 / 2}^{l} \alpha_{j-1 / 2}^{l}\right|\right) /\left(\alpha_{j+1 / 2}^{l}+\alpha_{j-1 / 2}^{l}\right)
\end{aligned}
$$




$$
\begin{aligned}
& g_{j}^{l}=\left\{\alpha_{j-1 / 2}^{l}\left[\left(\alpha_{j+1 / 2}^{l}\right)^{2}+\delta\right]+\alpha_{j+1 / 2}^{l}\left[\left(\alpha_{j-1 / 2}^{l}\right)^{2}+\delta\right]\right\} /\left[\left(\alpha_{j+1 / 2}^{l}\right)^{2}+\left(\alpha_{j-1 / 2}^{l}\right)^{2}+2 \delta\right] \\
& g_{j}^{l}=\min \bmod \left(2 \alpha_{j-1 / 2}^{l}, 2 \alpha_{j+1 / 2}^{l}, \frac{1}{2}\left(\alpha_{j+1 / 2}^{l}+\alpha_{j-1 / 2}^{l}\right)\right) \\
& g_{j}^{l}=S \cdot \max \left[0, \min \left(2\left|\alpha_{j+1 / 2}^{l}\right|, S \cdot \alpha_{j-1 / 2}^{l}\right), \min \left(\left|\alpha_{j+1 / 2}^{l}\right|, 2 S \cdot \alpha_{j-1 / 2}^{l}\right)\right] ; \\
& \quad S=\operatorname{sgn}\left(\alpha_{j+1 / 2}^{l}\right) .
\end{aligned}
$$

Here $\delta$ is a small parameter to prevent division by zero. In practical calculations $10^{-7} \leqslant \delta \leqslant 10^{-5}$ is a commonly used range.

MUSCL approach using an approximate Riemann solve. The numerical flux function $\tilde{F}_{j+1 / 2, k}$ for a MUSCL type approach, together with Roe type of approximate Riemann solver, for an upwind scheme as described in Yee [26] and Yee and Shinn [2] can be expressed as

$$
\begin{aligned}
\tilde{F}_{j+1 / 2, k}= & \frac{1}{2}\left\{\left(\frac{\xi_{x}}{J}\right)_{j+1 / 2}\left[F\left(U_{j+1 / 2}^{R}\right)+F\left(U_{j+1 / 2}^{L}\right)\right]+\left(\frac{\xi_{y}}{J}\right)_{j+1 / 2}\right. \\
& \left.\times\left[G\left(U_{j+1 / 2}^{R}\right)+G\left(U_{j+1 / 2}^{L}\right)\right]+\hat{R}_{j+1 / 2} \hat{\Phi}_{j+1 / 2} / J_{j+1 / 2}\right\} .
\end{aligned}
$$

The values $F\left(U_{j+1 / 2}^{R}\right)$ and $F\left(U_{j+1 / 2}^{L}\right)$ are the flux function $F$ evaluated at $U_{j+1 / 2}^{R}$ and $U_{j+1 / 2}^{L}$, respectively, with

$$
\begin{aligned}
& U_{j+1 / 2}^{R}=U_{j+1, k}-\frac{1}{4}\left[(1-\bar{\eta}) \widetilde{\Delta_{j+3 / 2}}+(1+\bar{\eta}) \widetilde{\widetilde{\Delta_{j+1 / 2}}}\right] \\
& U_{j+1 / 2}^{L}=U_{j, k}+\frac{1}{4}\left[(1-\bar{\eta}) \widetilde{\widetilde{\Delta_{j-1 / 2}}}+(1+\bar{\eta}) \widetilde{\Delta_{j+1 / 2}}\right],
\end{aligned}
$$

where $\bar{\eta}$ discussed below, is a parameter to control the spatial accuracy of the scheme. The limiters $\widetilde{\Delta_{j+1 / 2}}$ and $\widetilde{\Delta_{j+1 / 2}}$ can be expressed as

$$
\begin{aligned}
& \widetilde{\Delta_{j+1 / 2}}=\operatorname{minmod}\left(\Delta_{j+1 / 2}, \beta \Delta_{j-1 / 2}\right) \\
& \widetilde{\widetilde{\Delta_{j+1 / 2}}}=\operatorname{minmod}\left(\Delta_{j+1 / 2}, \beta \Delta_{j+3 / 2}\right)
\end{aligned}
$$

with

$$
\min \bmod (x, \beta y)=\operatorname{sgn}(x) \cdot \max \{0, \min [|x|, \operatorname{sgn}(x) \cdot \beta y]\}
$$

and $\beta=(3-\bar{\eta}) /(1-\bar{\eta})$, where $\Delta_{j+1 / 2}=U_{j+1, k}-U_{j, k}$. For $\bar{\eta}=-1, \widetilde{\Delta_{j+1 / 2}}$ and $\widetilde{\Delta_{j+1 / 2}}$ can be the same limiter as (9) except the arguments are now the $\Delta_{j \pm 1 / 2}$ instead of $\alpha_{j \pm 1 / 2}$.

The matrix $\hat{R}_{j+1 / 2}$ is the eigenvector of $A$ evaluated at some symmetric average of $U_{j+1 / 2}^{R}$ and $U_{j+1 / 2}^{L}$; i.e.,

$$
\hat{R}_{j+1 / 2}=R_{\xi}\left(U_{j+1 / 2}^{R}, U_{j+1 / 2}^{L}\right)
$$


and the elements of $\hat{\Phi}_{j+1 / 2}$ are

$$
\hat{\phi}_{j+1 / 2}^{l}=\psi\left(\hat{a}_{j+1 / 2}^{l}\right) \hat{\alpha}_{j+1 / 2}^{l}
$$

where again $\hat{a}_{j+1 / 2}^{l}$ and $\hat{\alpha}_{j+1 / 2}^{l}$ are evaluated at some symmetric average of $U_{j+1 / 2}^{R}$ and $U_{j+1 / 2}^{L}$ and

$$
\begin{aligned}
& \hat{a}_{j+1 / 2}^{l}=a_{\xi}^{l}\left(U_{j+1 / 2}^{R}, U_{j+1 / 2}^{L}\right) \\
& \hat{\alpha}_{j+1 / 2}^{l}=\hat{R}_{j+1 / 2}^{-1}\left(U_{j+1 / 2}^{R}-U_{j+1 / 2}^{L}\right) .
\end{aligned}
$$

Here the spatial order of accuracy pertaining to the scheme with the limiter not present (i.e., remove the tildes from Eqs. (10b), (10c)) is determined by the value of $\bar{\eta}$. For example, if $\bar{\eta}=-1$ the scheme is fully upwind and, if $\bar{\eta}=0$, it is Fromm's scheme. When $\bar{\eta}=\frac{1}{3}$ the scheme is third order and when $\bar{\eta}=1$, it is the regular three-point central difference scheme.

MUSCL approach using flux-vector splittings. The numerical flux function $\tilde{F}_{j+1 / 2, k}$ for a MUSCL-type approach, together with flux-vector splittings [8] referred as the TVD flux-vector splitting method in this paper can be expressed as

$$
\begin{aligned}
\tilde{F}_{j+1 / 2, k}= & \left(\frac{\xi_{x}}{J}\right)_{j+1 / 2}\left[F^{-}\left(U_{j+1 / 2}^{R}\right)+F^{+}\left(U_{j+1 / 2}^{L}\right)\right] \\
& +\left(\frac{\xi_{y}}{J}\right)_{j+1 / 2}\left[G^{-}\left(U_{j+1 / 2}^{R}\right)+G^{+}\left(U_{j+1 / 2}^{L}\right)\right],
\end{aligned}
$$

where $F^{ \pm}\left(U_{j+1 / 2}^{R, L}\right)$ are evaluated using either the Steger-Warming type $[16,18]$ or van Leer type $[17,18]$ flux-vector splittings. The vectors $U_{j+1 / 2}^{R}$ and $U_{j+1 / 2}^{L}$ are the same as in Eqs. (10b), (10c). The quantity $F^{-}\left(U_{j+1 / 2}^{R}\right)$ is the portion of the flux $F$ with negative eigenvalues evaluated at $U_{j+1 / 2}^{R}$.

The operations count between (6)-(9) and (10), (11) is within $30 \%$ for a perfect gas. However, due to an extra evaluation per dimension in the curve fitting between the left and right states in an equilibrium real gas for (10), (11), additional computation is required for the MUSCL approach. The slight advantage of (10), (11) over (6)-(9) is that (10), (11) can be spatially third-order accurate. However, experiences with the third-order case $\left(\bar{\eta}=\frac{1}{3}\right)$ do not show a very visible improvement over the second-order case for problems with discontinuities. Part of the reason is that all TVD-type schemes reduce to first order at points of extrema regardless of the order of accuracy at smooth regions. Also, because of the similarity in shock resolution and yet higher operations count for real gases and nonequilibrium flows of the MUSCL over the non-MUSCL approach using Roe type approximate Riemann solver, efforts are concentrated only on the nonMUSCL formulation. At present no outstanding advantages or disadvantages between these formulations for a perfect gas have been observed. Further investigation is required along this line before a clearer comparison can be drawn. 


\subsection{A Conservative Linearized Implicit Form for Unsteady and Steady-State Calculations}

To solve for $U^{n+1}$ in (5) one normally needs to solve a set of nonlinear algebraic equations iteratively. One way to avoid this is to linearize the implicit operator and solve the linearized form by other means. Following the same procedure as in Refs. [4-6], a conservative linearized alternating direction implicit (ADI) form of (5) for the numerical fluxes (6) and (10a) can be written as

$$
\begin{gathered}
{\left[I+\frac{\lambda^{\xi} \theta}{1+\omega} H_{j+1 / 2, k}^{\xi}-\frac{\lambda^{\xi} \theta}{1+\omega} H_{j-1 / 2, k}^{\xi}\right]^{n} E^{*}} \\
=-\frac{\lambda^{\xi}}{1+\omega}\left[\tilde{F}_{j+1 / 2, k}^{n}-\tilde{F}_{j-1 / 2, k}^{n}\right]-\frac{\lambda^{\eta}}{1+\omega}\left[\tilde{G}_{j, k+1 / 2}^{n}-\tilde{G}_{j, k-1 / 2}^{n}\right]+\frac{\omega}{1+\omega} \Delta \hat{U}_{j, k}^{n-1}, \\
{\left[I+\frac{\lambda^{\eta} \theta}{1+\omega} H_{j, k+1 / 2}^{\eta}-\frac{\lambda^{n} \theta}{1+\omega} H_{j, k-1 / 2}^{\eta}\right]^{n} E^{n}=E^{*},} \\
E^{n}=\Delta \hat{U}^{n} ; \quad \hat{U}^{n+1}=\hat{U}^{n}+E^{n},
\end{gathered}
$$

where for the finite volume formulation (6a)

and

$$
\begin{aligned}
H_{j+1 / 2, k}^{\xi} E^{*} & =\frac{1}{2}\left[\hat{\hat{A}}_{j+1, k} E_{j+1, k}^{*}+\hat{\hat{A}}_{j, k} E_{j, k}^{*}-\Omega_{j+1 / 2, k}^{\xi} E^{*}\right] \\
\hat{\hat{A}}_{j, k} & =\left[\left(\frac{\xi_{x}}{J}\right)_{j+1 / 2} A_{j, k}+\left(\frac{\xi_{y}}{J}\right)_{j+1 / 2} B_{j, k}\right] J_{j, k}
\end{aligned}
$$

$$
\hat{\hat{A}}_{j+1, k}=\left[\left(\frac{\xi_{x}}{J}\right)_{j+1 / 2} A_{j+1, k}+\left(\frac{\xi_{y}}{J}\right)_{j+1 / 2} B_{j+1, k}\right] J_{j+1, k} .
$$

Whereas for the pseudo-finite volume cf. Ref. [5]

$$
H_{j \pm 1 / 2, k}^{\xi} E^{*}=\frac{1}{2}\left[\hat{A}_{j \pm 1, k} E_{j \pm 1, k}^{*}-\Omega_{j \pm 1 / 2, k}^{\xi} E^{*}\right]
$$

is used, and $\Omega_{j+1 / 2, k}^{\xi} E^{*}, \Omega_{j, k+1 / 2}^{\eta} E$ can be taken as

$$
\begin{aligned}
\Omega_{j+1 / 2, k}^{\xi} E^{*} & =R_{j+1 / 2, k} \operatorname{diag}\left[\psi\left(a_{j+1 / 2}^{l}\right)\right] R_{j+1 / 2, k}^{-1}\left(E_{j+1, k}^{*}-E_{j, k}^{*}\right) \\
\Omega_{j, k+1 / 2}^{\eta} E & =R_{j, k+1 / 2} \operatorname{diag}\left[\psi\left(a_{k+1 / 2}^{l}\right)\right] R_{j, k+1 / 2}^{-1}\left(E_{j, k+1}-E_{j, k}\right) .
\end{aligned}
$$

Here $\hat{A}_{j+1, k}, \hat{B}_{j, k+1}$ are Jacobians of $\hat{F}$ and $\hat{G}$ evaluated at $(j+1, k)$ and $(j, k+1)$. The value $E_{j, k}^{n}=\hat{U}_{j, k}^{n+1}-\hat{U}_{j, k}^{n}$. The expression $\operatorname{diag}\left(z^{l}\right)$ denotes a diagonal matrix with diagonal elements $z^{l}$.

The nonconservative linearized implicit form suitable for steady-state calculations [4] is also considered. Numerical study indicated that the latter form appears to be slightly less efficient in terms of convergence rate than the linearized conservative form (12). This conservative linearized implicit operator as well as the nonconservative linearized implicit operator (both suggested in Ref. [4]) for $\theta=1, \omega=0$ was 
rediscovered and renamed two years later by Barth [27] as the approximate Jacobian linearization. To compute $(12 \mathrm{~g}),(12 \mathrm{~h})$, a triple matrix multiplication of dimension $(4 \times 4)$ has to be performed at every grid point. For steady-state applications, one can simplify $(12 \mathrm{~g}),(12 \mathrm{~h})$

$$
\begin{aligned}
& \Omega_{j+1 / 2, k}^{\xi} E=M_{\xi} I\left(E_{j+1, k}-E_{j, k}\right) \\
& \Omega_{j, k+1 / 2}^{\eta} E=M_{\eta} I\left(E_{j, k+1}-E_{j, k}\right) .
\end{aligned}
$$

The scalar values $M_{\xi}$ and $M_{\eta}$ are the spectral radii of $\Omega_{j+1 / 2, k}^{\xi}$ and $\Omega_{j, k+1 / 2}^{\eta}$ :

$$
\begin{aligned}
& M_{\xi}=\max _{l} \psi\left(a_{j+1 / 2}^{l}\right) \\
& M_{\eta}=\max _{l} \psi\left(a_{k+1 / 2}^{l}\right)
\end{aligned}
$$

and $I$ is the identity matrix. Note that (13a), (13b) involve scalar multiplication only. The solution using (13) is still second-order (or third-order) accurate after it reaches steady state. Other linearized implicit forms can be found in Refs. [4-6].

\subsection{General Assumptions and Limitations on the Numerical Studies}

The present study is by no means an exhaustive investigation. There are additional elements and parameters (other than the ones considered here) in the algorithm itself as well as in the physical problem, such as flow type and geometric complexity, that can affect or interfere with the performance of the numerical scheme. Even within the numerical issues listed in Section I, the study is limited to a sampling of the parameter range for the time-step size or CFL number and the form of $\delta_{1}$ in (7b). In particular, various strategies to speed up and stabilized the start-up solution from freestream conditions for steady computations have not been investigated. What is discussed here is intended to give interested readers some guideline for the use of the algorithm. All of the numerical studies discussed in the subsequent sections rely on the following assumptions and considerations:

1. Although we recommend the finite volume formulation (6), the results obtained in this report used a slightly different formulation than (6). In particular, three formulations (hereafter referred to as the pseudo finite volume formulations) for the non-MUSCL schemes were investigated and are

$$
\begin{aligned}
\tilde{F}_{j+1 / 2, k}= & \frac{1}{2}\left[\left(\xi_{x}\right)_{j+1 / 2}\left(F_{j, k}+F_{j+1, k}\right)+\left(\xi_{y}\right)_{j+1 / 2}\left(G_{j, k}+G_{j+1, k}\right)\right. \\
& \left.+R_{j+1 / 2} \Phi_{j+1 / 2}\right] / J_{j+1 / 2}
\end{aligned}
$$

with the corresponding quantities $\left(\xi_{x}\right)_{j+1 / 2}, J_{j+1 / 2}$ and $\left(k_{1}\right)_{j+1 / 2}$ of Eqs. (6b), (6c) expressed as

$$
\begin{aligned}
& \left(\xi_{x}\right)_{j+1 / 2}=\frac{1}{2}\left[\left(\xi_{x}\right)_{j+1, k}+\left(\xi_{x}\right)_{j, k}\right] ; \quad J_{j+1 / 2}=\frac{1}{2}\left[J_{j+1, k}+J_{j, k}\right], \\
& \left(k_{1}\right)_{j+1 / 2}=\frac{\left(\xi_{x}\right)_{j+1 / 2}}{\sqrt{\left(\xi_{x}\right)_{j+1 / 2}^{2}+\left(\xi_{y}\right)_{j+1 / 2}^{2}}},
\end{aligned}
$$


and

$$
\tilde{F}_{j+1 / 2, k}=\frac{1}{2}\left[\left(\xi_{x} F+\xi_{y} G\right)_{j, k}+\left(\xi_{x} F+\xi_{y} G\right)_{j+1, k}+R_{j+1 / 2} \Phi_{j+1 / 2}\right] / J_{j+1 / 2}
$$

and

$$
\widetilde{F}_{j+1 / 2, k}=\frac{1}{2}\left[\hat{F}_{j+1, k}+\hat{F}_{j, k}+R_{j+1 / 2} \Phi_{j+1 / 2} / J_{j+1 / 2}\right] .
$$

Here $J_{j+1 / 2}$ and $k_{1}$ in Eqs. (15) and (16) are the same as (14b), (14c). For highly skewed grids and nonuniform flows, Eqs. (6) and (14) are preferred over (15) and (16). However, (14) and (15) do not preserve freestream whereas Eqs. (6) and (16) do. All of the results present in Section V use (15). One of the blunt-body cases was rerun with Eqs. (6) and (14)-(16) and no noticeable difference was observed. We expect all of the conclusions on the behavior of (14)-(16) to be carried over to Eq. (6), since all of the examples use mildly clustered yet quite regular and nearly orthogonal grids.

In two dimensions the present pseudo finite volume formulations can be made "truly" finite volume by a slight modification of Eqs. (14)-(16); i.e., on the treatment of $k_{1}$ and $J_{j+1 / 2}$. However, the situation is different in three dimensions where finite volume formulations depart from finite difference formulations. See Ref. [28] for a discussion.

2. The numerical results and conclusions are for the non-MUSCL approach and for the particular flow type and geometry specified with a sampling of a narrow range of Mach numbers and time steps. Results for viscous flow calculations are based on the shock wave dominated thin-layer Navier-Stokes equations for laminar flows.

3. The numerical boundary condition treatments are the same as in Refs. $[5,29,30]$ for the inviscid flow and as in Ref. [21] for the viscous flows. Studies [31] showed that proper treatment of numerical boundary conditions has a major impact on the stability and convergence rate of the scheme. Therefore the types of boundary condition treatment used here reflect on the performance of the stability, accuracy, and convergence rate of the present algorithm.

4. For steady-state computations, the convergence rate not only depends on all of the elements and parameters (to be discussed shortly), but more importantly also on the type of grid associated with the computation. Studies show that, in general, a coarse nearly uniform orthogonal grid converges 1-3 times faster than similar finer grids, and possibly an order of magnitude or more faster than highly clustered or skewed grids. What will be presented in Section V represents fairly uniform to mildly clustered grids. Most of the grids used for the numerical study were not very coarse; thus the number of iterations quoted is naturally higher than its coarse grid counterpart.

5. For the non-interfering blunt-body flows, the convergence rate and behavior of the symmetric and upwind TVD-type schemes are very similar. However, for the interfering blunt-body flows containing slip or shear surfaces, the 
upwind scheme produces sharper weak solutions. Consequently, all of the illustrations and conclusions discussed in this paper are for the upwind scheme using limiter (9c). Other limiters can produce sharper discontinuities but are not as robust as limiter (9c). See Section V or Ref. [12] for a discussion.

6. Research on real gas effects on the performance of the proposed scheme is only in the preliminary stage. All of the illustrations and conclusions for real gases are for inviscid non-interfering blunt-body flows. Study of viscous real gas flows is in progress.

7. For steady-state computations using the backward Euler time differencing $(\theta=1, \omega=0)$, a local time stepping procedure similar to [30,32] was used. However, in comparing the convergence rate with the three-point backward differentiation time differencing $\left(\theta=1, \omega=\frac{1}{2}\right)$ for the viscous flows, a constant time step was used.

Other issues such as reducing ADI factoring error, using multigrid, relaxation, or conjugate gradient methods as an alternative to ADI, using local grid refinement to enhance resolution, etc., which are also sources of consideration to algorithm (6)-(16), are not considered here. These issues and the development of better algorithms are the subject of on-going research.

\section{Enhancement of Stability and Convergence Rate for Hypersonic Flows}

In Ref. [1], some elements and parameters which can affect the stability and convergence rate in high Mach number cases but have negligible effect in low Mach number cases for steady-state inviscid blunt-body flows were identified. The current study indicated that the same elements and parameters can affect the stability and convergence rate at hypersonic speeds for viscous computations as well. They are: (1) the choice of the entropy correction parameter $\delta_{1}$, (2) the choice of the dependent variables on which the limiters are applied (related to proper scaling of the eigenvectors for high speed flows), and (3) the prevention of unphysical solutions during the initial transient stage. Our study indicates that these elements can also improve the stability of unsteady as well as steady hypersonic flows.

1. For Mach numbers ranging from 1.2 to 15 , numerical experiments for oneand higher dimensional unsteady flows containing unsteady shocks show that the second-order explicit TVD schemes $[29,12,11]$ are insensitive to the entropy correction for $0 \leqslant \delta_{1} \leqslant 0.1$. In most cases $\delta_{1}=0$ was used. For $0.1 \leqslant \delta_{1} \leqslant 0.25$, there is a possibility of improving stability in the sense of allowing a higher CFL number at the expense of a slight smearing of the discontinuities. However, for unsteady complex shock wave interactions, a small positive $\delta_{1}$ or a variable $\delta_{1}$ (to be discussed) can help stabilize the time-accurate implicit algorithm (12).

For subsonic to low supersonic steady-state NACA 0012 airfoil computations $[5,6]$, the resolution of the shock waves was found to be quite insensitive to 
$0.1 \leqslant \delta_{1} \leqslant 0.125$ and a constant value seems to be sufficient. However, for hypersonic flows, especially for blunt-body flows, a constant $\delta_{1}$ or a variable $\delta_{1}$ suggested by Harten and Hyman [33] was found to be insufficient, but a variable $\delta_{1}$ depending on the spectral radius of the Jacobian matrices of the fluxes is very helpful in terms of stability and convergence rate. In fact, a proper choice of the entropy parameter $\delta_{1}$ for higher Mach number flows not only helps in preventing nonphysical solutions but can act as a control of the convergence rate and of the sharpness of shocks and slip surfaces (or shear layer in viscous flows). The smaller the $\delta_{1}$ that is used, the slower is the convergence rate. The larger the $\delta_{1}$ that is being used, the larger is the numerical dissipation being added. However, $\delta_{1}$ cannot be arbitrarily large.

For the present blunt-body steady-state calculations with Mach numbers $M>4$, the initial flow conditions at the wall are obtained using the known wall temperature in conjunction with pressures computed from a modified Newtonian expression [34]. Also, for implicit methods a slow start-up procedure from initial conditions [30] is necessary. Most importantly, experience indicates that if one sets $\delta_{1}$ in Eq. (7b) as a function of the velocity and sound speed, i.e.,

$$
\begin{aligned}
& \left(\delta_{1}\right)_{j+1 / 2}=\tilde{\delta}\left(\left|u_{j+1 / 2}\right|+\left|v_{j+1 / 2}\right|+c_{j+1 / 2}\right) \\
& \left(\delta_{1}\right)_{k+1 / 2}=\tilde{\delta}\left(\left|u_{k+1 / 2}\right|+\left|v_{k+1 / 2}\right|+c_{k+1 / 2}\right)
\end{aligned}
$$

with $0.05 \leqslant \tilde{\delta} \leqslant 0.25$, then blunt-body flows for $4 \leqslant M \leqslant 25$ appear to be stabilized and nonphysical solutions are less likely to occur. Equation (17) is written in Cartesian coordinates. In the case of generalized coordinates, the $u$ and $v$ should be replaced by the contravariant velocity components, and one half of the sound speed would be from the $\xi$-direction and the other half would be from the $\eta$-direction. For implicit methods, it is very important to use (17) in $\psi(z)$ on both the implicit and explicit operators, since in a two-dimensional hypersonic flow field consisting of a mixture of subsonic and supersonic regions with Mach numbers ranging from 0 to hypersonic speeds, an entropy parameter like (17) is nonzero in all of the regions. The entropy parameter (17) seems to work well for blunt-body flows but whether this is also the right choice for configurations other than a blunt-body shape is an open question.

For unsteady hypersonic blunt body complex shock wave interactions, the entropy parameter (17) can help stabilize the time-accurate implicit algorithm. For most of the viscous and inviscid calculations shown, unless otherwise indicated, $\tilde{\delta}$ is set to 0.125 .

2. Higher order TVD schemes in general involve limiter functions. However, there are options in choosing the types of dependent variables when applying limiters for systems of hyperbolic conservation law, in particular, for systems in generalized coordinates. The choice of the dependent variables on which limiters are applied can affect the stability and convergence process. In particular, due to the nonuniqueness of the eigenvectors $R_{j+1 / 2}$, the choice of the characteristic variables 
on which the limiters are applied play an important role in the stability and convergence rate as the Mach number increases. This is directly related to proper scaling of the eigenvectors for high speed flows. For moderate Mach numbers, the different choices of the eigenvectors have a negligible effect on the stability and convergence rate. However, for large Mach number cases, the magnitudes of all the variables at the jump of the bow shock are not the same. In general, the jumps are much larger for the pressures than for the densities or total energy. Studies indicated that employing the form $R_{j+1 / 2}$ such that the variation of the $\alpha$ are of the same order of magnitude as the pressure would be a good choice for hypersonic flows. The form similar to the one used by Gnoffo [35] or Roe and Pike [36] can improve the convergence rate over the ones used in Refs. [37,38]. In all of the computations shown, the form $R_{j+1 / 2}$ used is the same as in Refs. [37, 38] except for an extra factor of $1 / c_{j+1 / 2}^{2}$. With this extra factor the variation of the $\alpha$ are in fact proportional to the pressure. Other forms of $R_{j+1 / 2}$ have not been investigated. With this type of scaling, the stability criterion is less restricted even for unsteady hypersonic speeds.

3. Due to the large gradients and to the fact that the initial conditions are far from the steady-state physical solution, the path used by the implicit method can go through states with negative pressures if a large time step is employed. A convenient way to overcome this difficulty is to fix a minimum non-negative allowed value for the density and the pressure. With this safety check, the scheme allows a much larger time step and converges several times faster.

In addition, since the Roe's average state allows the square of the average sound speed $c_{j+1 / 2}^{2}$ to lie outside the interval between $c_{j}^{2}$ and $c_{j+1}^{2}$ for equilibrium real gases, the average state $c_{j+1 / 2}^{2}$ might be negative even though $c_{j}^{2}$ and $c_{j+1}^{2}$ are positive during the transient stage when the initial conditions are far from the steady-state physical solution. In this case, we replace $c_{j+1 / 2}^{2}$ by $\max \left(c_{j+1 / 2}^{2}, \min \left(c_{j}^{2}, c_{j+1}^{2}\right)\right)$. This latter safety check is in particular helpful for the symmetric TVD algorithm (7) but not necessary for the upwind algorithm (9).

\section{Behavior of the Algorithm with Different Temporal Differencing}

It is emphasized here that since the method (12) is written in the "delta" formulation, either the backward Euler (first-order) or the three-point backward differentiation (second-order) time discretizations require the same amount of storage and a similar operation count. Therefore, the main consideration between the two timedifferencing methods is their relative stability and convergence rate.

Inviscid unsteady flows. For inviscid unsteady flows, the explicit TVD-type methods $[29,12,11]$ are more efficient than the second-order implicit method (12). Unless the inviscid problem is stiff, there is no advantage of employing an implicit method for inviscid unsteady flows. 
Inviscid steady flow. The backward Euler implicit method has a better stability and convergence rate than the three-point backward differentiation implicit method. Also a local time-stepping procedure $[30,32]$ can speed up the convergence rate for the former time-differencing method whereas the same procedure has little effect on the convergence rate when compared with a fixed time step procedure for the latter time-differencing method.

Viscous unsteady flows. Computations on the unsteady viscous flows mainly use the second-order time differencing since a larger time step can be used (but still remain time-accurate at least slightly away from the wall) compared with the temporally first-order implicit method. Due to the highly clustered viscous grid used in contrast to their inviscid counterpart, solving a viscous unsteady complex shock interaction using an explicit TVD-type method is not practical due to its inherent time step restriction. In certain cases, the time step might be an order of magnitude smaller than the implicit counterpart. A more detailed illustration of unsteady viscous hypersonic blunt-body flows with an impinging shock is reported in Ref. [22].

Viscous steady flows. At present there is no detailed viscous steady flow study comparing the first-order time differencing using a local time-stepping approach with the second-order time differencing using a constant time step approach. But the general trend is that the second-order time differencing has slightly better stability and convergence rate than the former one. In particular, a summary using a sequence of fixed time step approaches comparing the two time-differencing algorithms is discussed in Section V.

\section{Numerical RESULTS}

The various numerical aspects discussed in Sections III-IV are evaluated in part by experience gained from the one-dimensional shock tube results [11], from twodimensional subsonic, transonic, supersonic, and hypersonic speed flows results $[1,4-6,12]$ and in part by a variety of two-dimensional steady and unsteady, viscous and inviscid hypersonic blunt-body flow computations in this section. Some of the numerical studies are compared with shock-fitting and some are compared with experiments when available. Some of the more detailed computation of the physics of these problems can be found in Refs. [21, 22]. Six types of blunt-body test cases are illustrated in Figs. 1-11. Test cases 1 and 2 are inviscid, perfect and real gas, non-interfering blunt-body flows. Test case 3 is a steady inviscid, perfect gas blunt-body flow with an impinging shock. Test cases $4-6$ are viscous steady and unsteady perfect gas blunt-body flows with and without impinging shocks.

Comparison among the various linearized implicit methods. For the implicit operator, numerical experiments show that the linearized conservative form (12) converges slightly faster (while requiring almost identical CPU time per time step) 
than the linearized nonconservative form $[37,4]$ for both viscous and inviscid flows. It appears also that when the freestream Mach number increases, the convergence rate of the linearized conservative form (12) is better than a simplified version which replaces $\Omega_{j+1 / 2, k}^{\xi}$ and $\Omega_{j, k+1 / 2}^{\eta}$ of $(12 \mathrm{~g}),(12 \mathrm{~h})$ by $\max _{l} \psi\left(a_{j+1 / 2}^{l}\right)$ and $\max _{l} \psi\left(a_{k+1 / 2}^{l}\right)$ times the identity matrix (Eq. (13)). This is especially true for viscous flow computations. Due to the experience gained from the transonic and the inviscid hypersonic study, no detailed computations using the linearized nonconservative form were performed for viscous steady flow. All of the results and discussions for the viscous computations are based on the conservative linearized form.

Another area of investigation is that for viscous computations, the Jacobian of the viscous terms in the implicit operators are rather expensive to compute. For unsteady flows, these terms are needed to maintain the spatial order of accuracy. Whether the omission of these terms has a major impact on the stability and convergence rate of the algorithm for steady-state calculations is not known. Therefore, an investigation has been made on the difference in the convergence rate for the algorithm with or without the viscous terms in the implicit operator. A brief summary is included in one of the following subsections.

Choice of limiters. Unlike flows with transonic and low supersonic shock waves, problems containing strong hypersonic shock waves are more sensitive to the treatment of limiters. Using the more diffusive limiter (7c) or (9c) turns out to be more stable than other more compressive limiters. In terms of shock resolution for both the symmetric and upwind TVD-type of schemes, the sequences written in Eqs. $(7 c)-(7 e)$ and $(9 c)-(9 g)$ are in order of increasing accuracy. On the other hand, these sequences are in order of decreasing stability and convergence rate. The more compressive limiters like (9f) and (9g) have a very low stability and slow con-

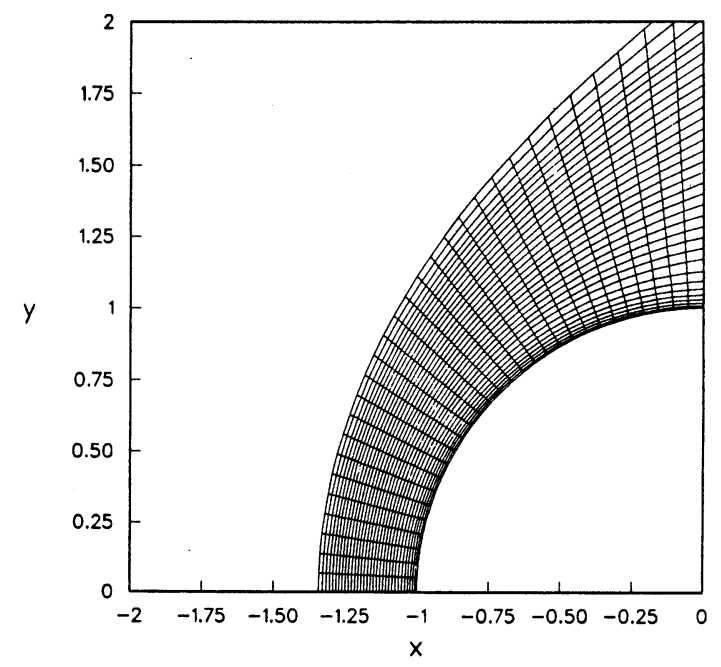

Fig. 1. The $31 \times 33$ grid. 
vergence rate for steady flows. The same conclusion applies for unsteady flows where the more compressive limiters have a very restricted time step limit. From our experiences, it is not advisable to use (9f) and $(9 \mathrm{~g})$ for complex steady shock wave interactions. In particular, limiter $(9 \mathrm{~g})$ should be used only for the linear fields (i.e., for the $u$ and $v$ characteristic fields in the $x$ - and $y$-direction, respectively). See Ref. [12] for more details.

Convergence rate of explicit and implicit TVD-type schemes for real gas flows. The five different second-order TVD methods previously studied [11] in one dimension yield very similar shock resolution for the blunt-body (noninterfering case) problem. In particular, for an inviscid blunt-body flow in the hypersonic equilibrium real gas range, the explicit second-order Harten and Yee, and Yee-Roe-Davis type TVD schemes $[6,24,25]$ using the generalized
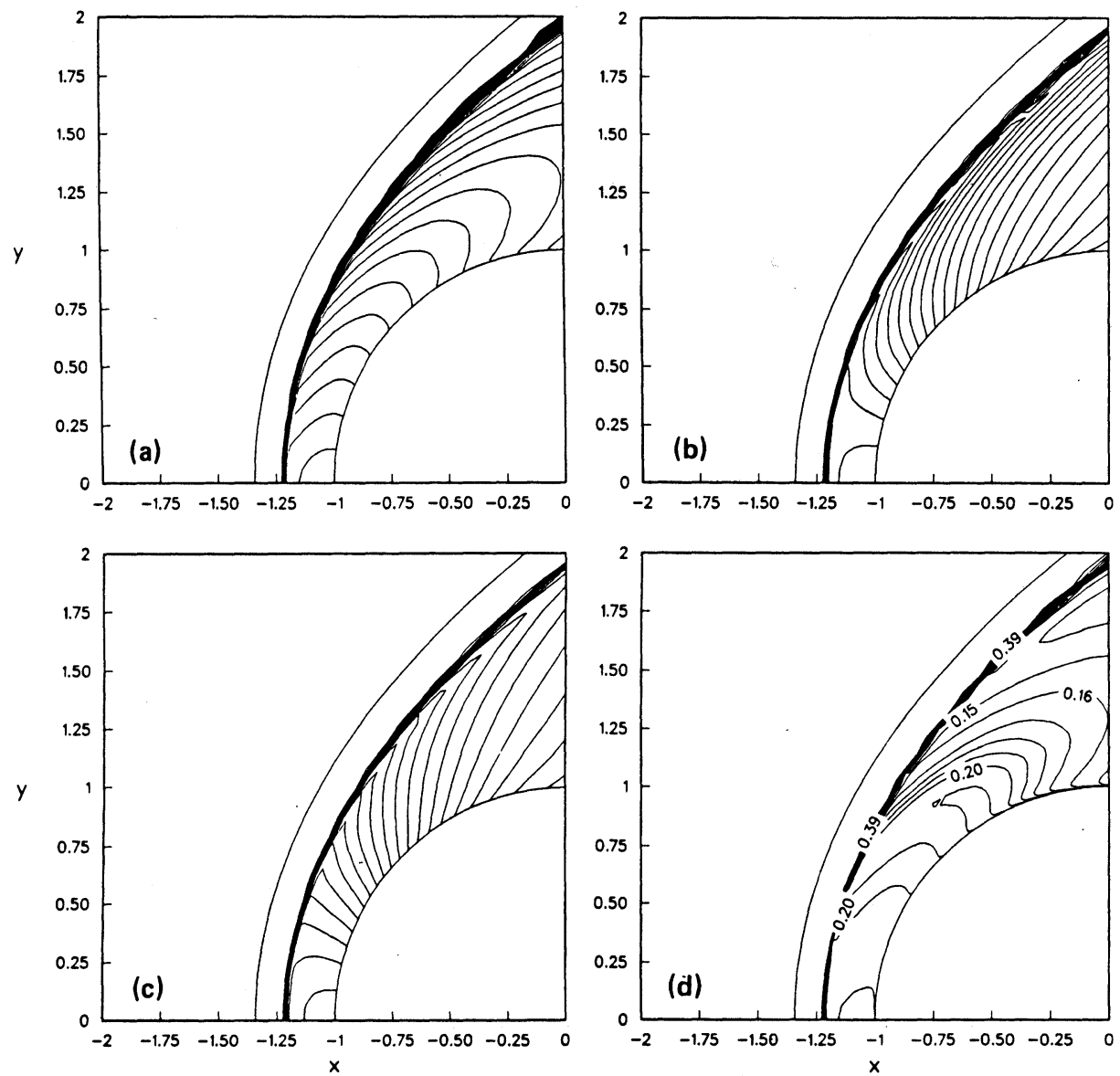

FIG. 2. The Mach contours (a), density contours (b), pressure contours (c), and $\kappa$ (d) computed by the implicit scheme (12) $(\theta=1, \omega=0)$ for an equilibrium real gas with $M_{\infty}=15$. 
approximate Riemann solver [13] produce similar shock-resolution but converge slightly faster (with comparable CPU time per time step) then an explicit secondorder van Leer type scheme using the generalized van Leer flux-vector splitting [11].

The freestream conditions for the current study are $M_{\infty}=15$ and 25 , $p_{\infty}=1.22 \times 10^{3} \mathrm{~N} / \mathrm{m}^{2}, \rho_{\infty}=1.88 \times 10^{-2} \mathrm{~kg} / \mathrm{m}^{3}$, and $T_{\infty}=226 \mathrm{~K}$. Figure 1 shows half of the $61 \times 33$ grid used for the blunt-body problem. For the $M_{\infty}=25$ case, the shock standoff distance is at approximately 14 points from the wall on the symmetry axis. The relaxation procedure for the explicit methods employs a secondorder Runge-Kutta time discretization [1] with a CFL of 0.5 (solution not shown). The parameter $\tilde{\delta}$ is set to a constant value of 0.15 . Pressure and Mach number contours (compared with shock-fitting solutions) converge and stabilize after
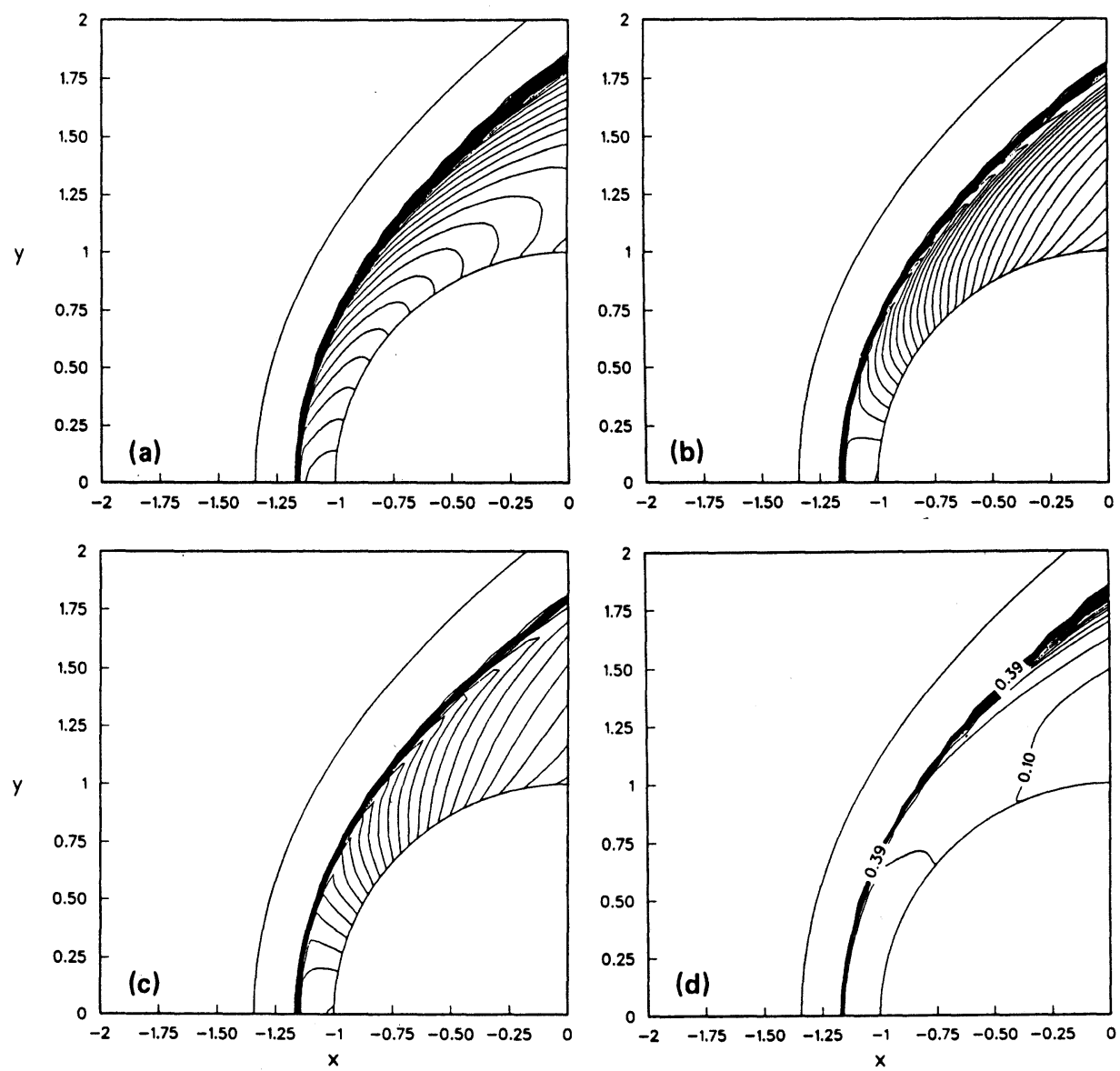

Fig. 3. The Mach contours (a), density contours (b), pressure contours (c), and $\kappa$ (d) computed by the implicit scheme (12) $(\theta=1, \dot{\omega}=0)$ for an equilibrium real gas with $M_{\infty}=25$. 

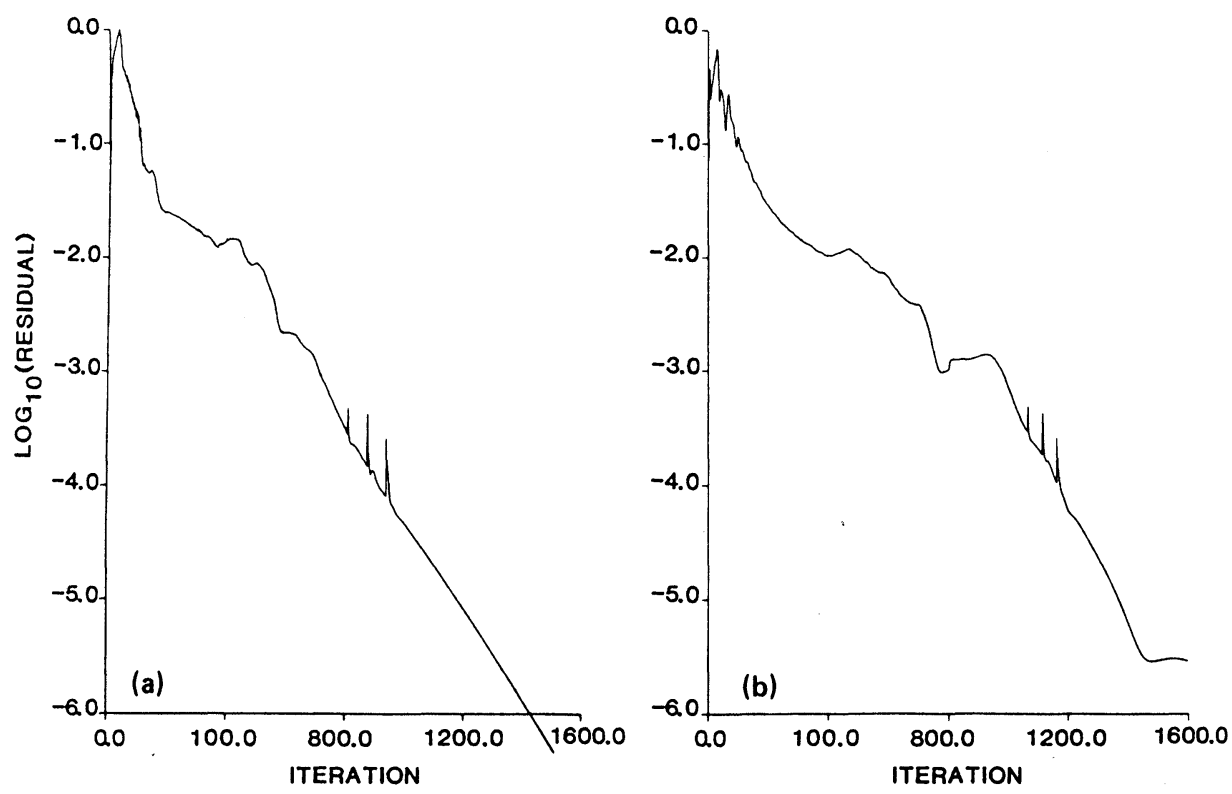

FIG. 4. Comparison of the $L_{2}$-norm residual of a linearized conservative implicit operator (a) and a linearized nonconservative implicit operator (b) for an equilibrium real gas with $M_{\infty}=25$.
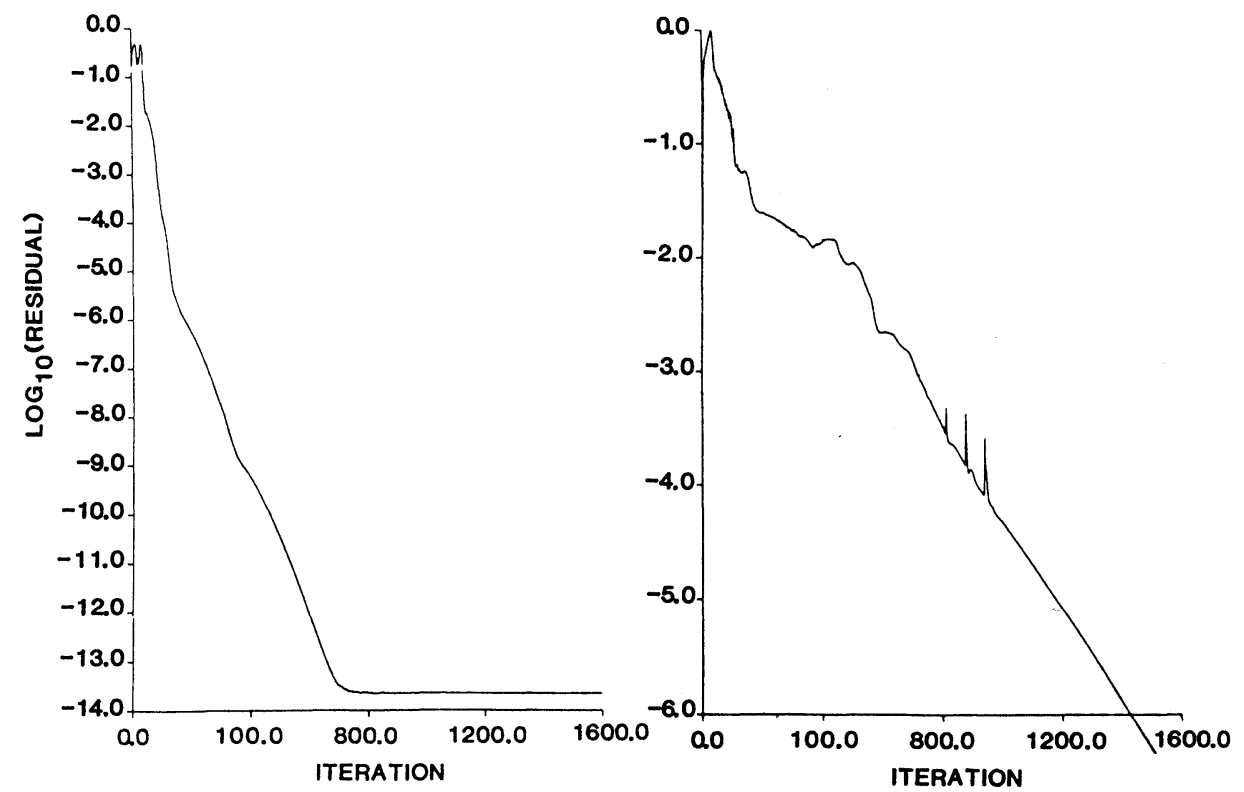

Fig. 5. Comparison of the $L_{2}$-norm residual of a perfect gas (left) and real gas (right) computed by the scheme (12) $(\theta=1, \omega=0)$ with $M_{\infty}=25$. Note that the scale of the ordinate for the perfect gas and the real gas is not the same. 
$3000-4000$ steps but the convergence rate is much slower for the density (with a 2-3 order of magnitude drop in $L_{2}$-norm residual). The bow shock is captured in two to three grid points. The curve fits of Srinivasan et al. [39] are used to generate the thermodynamic properties of the gas.

The same flow condition was tested on the implicit scheme (12) and the convergence rate was found to be many times faster. Figures 2 and 3 show the Mach number, density, pressure, and $\kappa$ contours computed by the linearized conservative ADI form of the upwind scheme (12) with the first-order backward Euler $(\theta=1$ and $\omega=0$ ) for Mach numbers 15 and 25. Figure 4 shows the slight advantage of the convergence rate of the linearized conservative implicit scheme (12) over the linearized nonconservative implicit scheme (with $\theta=1, \omega=0$ and a fixed CFL of 15) suggested in Ref. $[37,4]$. The convergence rate and shock resolution for the symmetric TVD-type scheme (12) behave similarly. For $M_{\infty}=15$ case, the $L_{2}$-norm residual stagnated after a drop of four orders of magnitude.

In general, for a perfect gas with $10 \leqslant M_{\infty} \leqslant 25$ and a not highly clustered grid, steady-state solutions can be reached in 600-800 steps with 12 orders of magnitude drop in the $L_{2}$-norm residual. However, the convergence rate is many times slower for the real gas counterpart. Figure 5 shows the convergence rate for a perfect gas

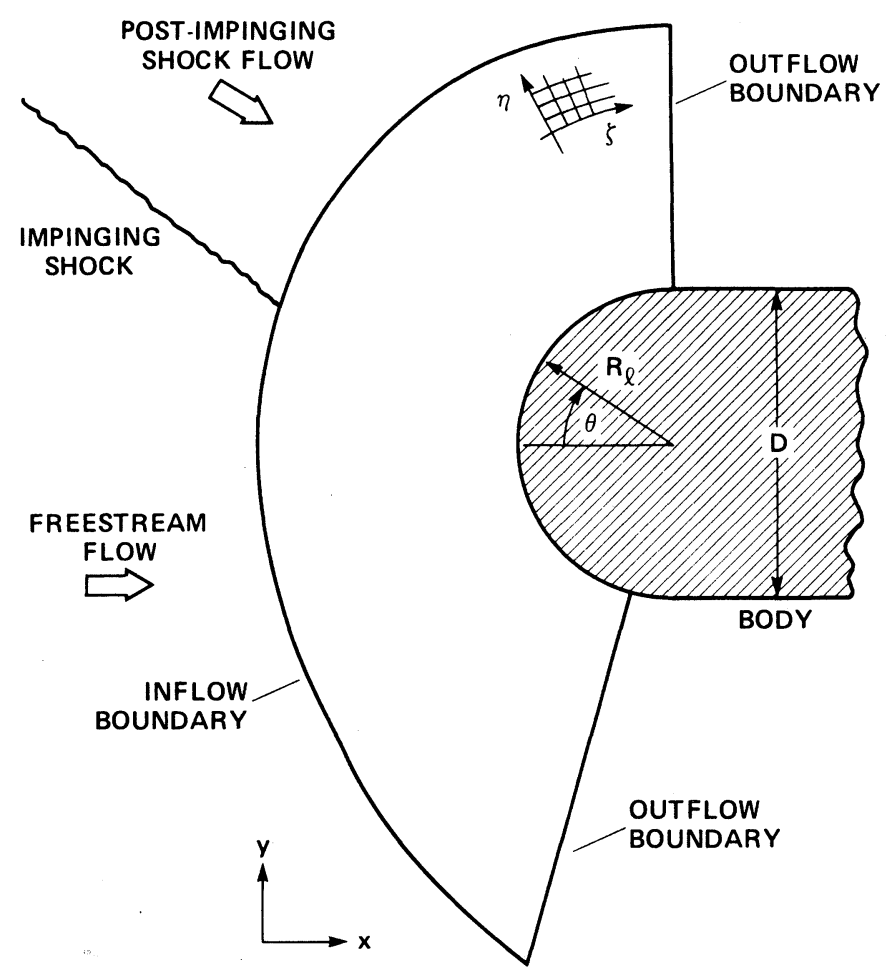

FIG. 6. Schematic of the computational domain for a blunt body flow with an impinging shock. 
compared with a real gas computation with a fixed CFL of 50. Note that the scale of the ordinates used in Fig. 5 for the perfect gas and the real gas are not the same. The freestream conditions for the real gas study are the same as Fig. 3. An important observation for the behavior of the convergence rate for the Mach 15 real gas case is that the discontinuities of the thermodynamic derivatives which exist in the curve fits of Srinivasan et al. [39] might be the major contributing factor. This is evident from Figs. 2d and $3 d$ and from a comparison with the convergence rate for the perfect gas. Another contributing factor is that the curve fits are accurate only for temperatures up to $6000 \mathrm{~K}$. Since the temperature in this case is slightly above $6000 \mathrm{~K}$, there is an uncertainty in the accuracy of the computed results. Further improvement of the existing curve fitting procedure is needed in order to access the "true" difference in convergence rate between perfect and real gases.

Inviscid impinging shock computations. Figures 6 and 7 show the schematic of the computational domain, the Mach contours, and the $L_{2}$-norm residual computed by the implicit upwind scheme (12) (with $\theta=1, \omega=0$ ) of an inviscid shockon-shock interaction on a blunt body with radius $R_{l}$ and thickness $D=2 R_{l}$ in the

GRID

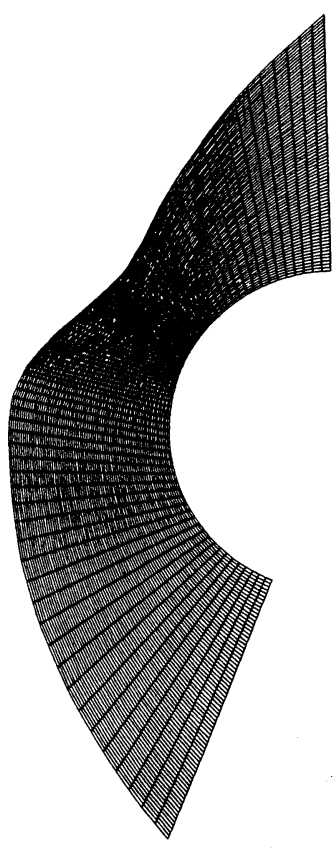

MACH CONTOURS

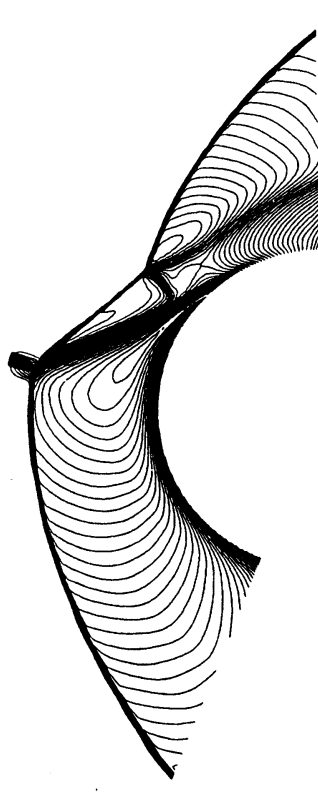

CONVERGENCE RATE

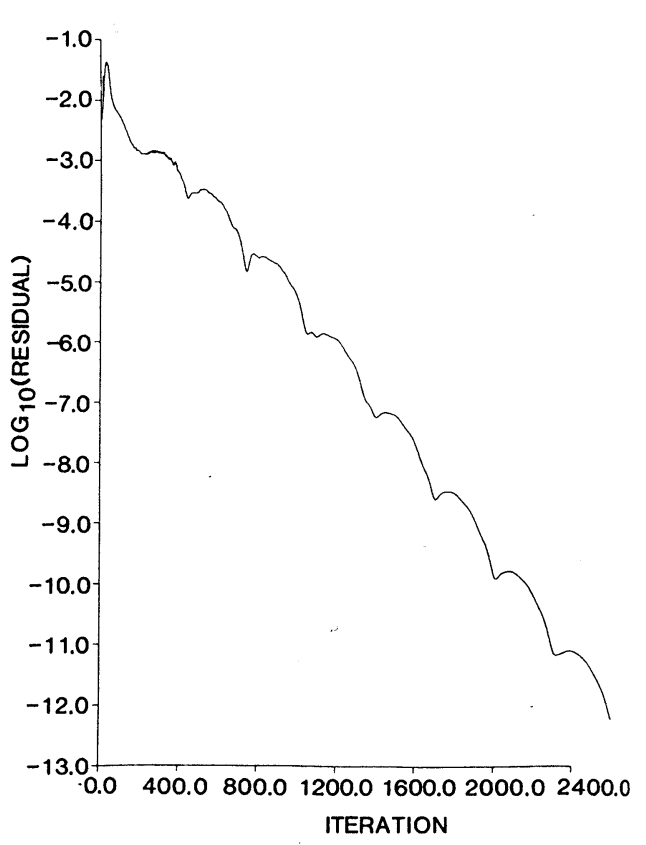

FIG. 7. Two-dimensional inviscid steady blunt-body flow with an impinging shock computed by the implicit scheme (12) $(\theta=1, \omega=0)$ for a perfect gas with $M_{\infty}=4.6$. 
low hypersonic range $4 \leqslant M_{\infty}<8$. Higher inviscid hypersonic Mach number $8 \leqslant M_{\infty} \leqslant 30$ computations using the proposed scheme are also possible but are not shown here. Some viscous and inviscid studies on flow fields of this type were reported in Refs. [34, 40-42]. This flow field is typical of what may be experienced by the inlet cowl of a hypersonic aerodynamic vehicle. The freestream conditions for this flow field are the freestream Mach number $M_{\infty}=4.6$, the freestream temperature $T_{\infty}=167 \mathrm{~K}$, and $\gamma=1.4$ for a perfect gas. An oblique shock with an angle of $20.9^{\circ}$ relative to the free stream impinges on the bow shock. Various types of interactions occur depending on where the impingement point is located on the bow shock. As shown by the Mach contours ranging from 0 to 4.55 in increments of 0.05 , the impinging shock has caused the stagnation point to move away from its undisturbed location at the symmetry line. The surface pressures at the new stagnation point can be several times larger than those at the undisturbed location of the stagnation point. In addition, a slip surface emanates from the bow shock and impinging shock intersection point and is intercepted by a shock wave which starts at the upper kink of the bow shock. The interacting shock waves and slip surfaces are confined to a very small region and must be captured accurately by the numerical scheme if the proper surface pressures are to be predicted correctly. The $77 \times 77$ grid used and the convergence rate computed by the implicit scheme (12) are shown in Fig. 7. Though the pattern of the flow is significantly more complicated than for the previous cases, the convergence rate remains quite satisfactory.
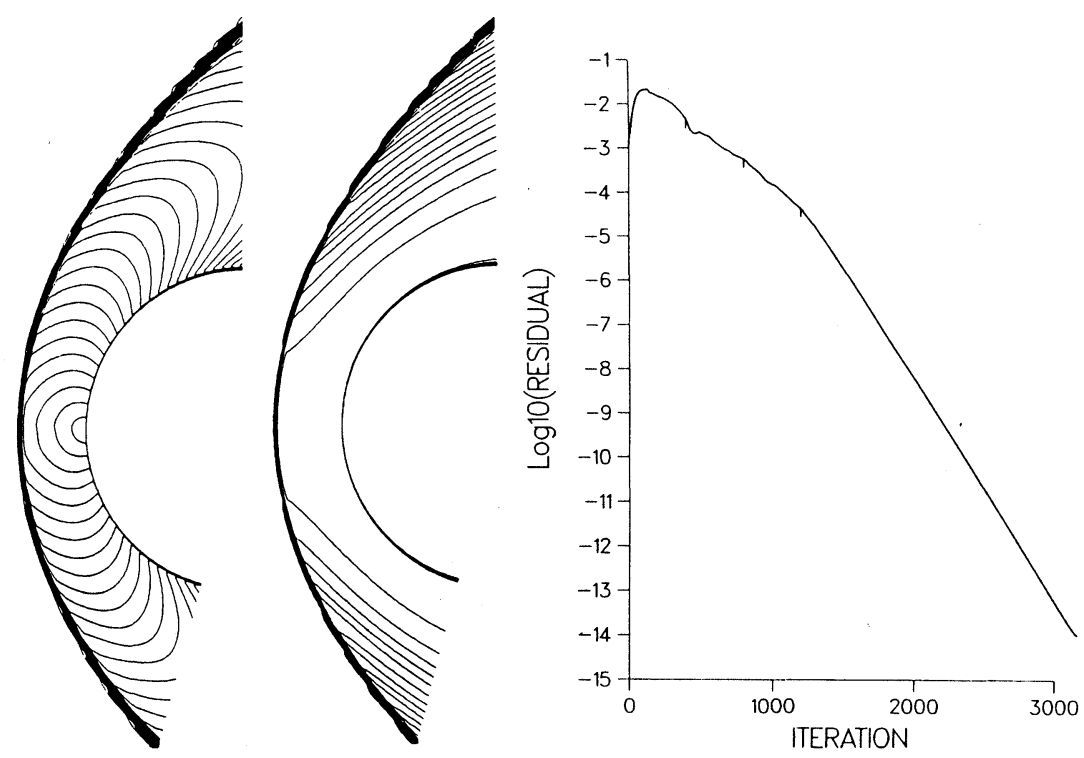

Fig. 8. Mach contours, entropy contours, and residual history for the steady viscous blunt body flow computed by algorithm (12) $\left(\theta=1, \omega=\frac{1}{2}\right.$, full matrix form) with $M_{\infty}=8.03, \operatorname{Re}_{D}=387,750, \gamma=1.4$, and laminar flow. 
As shown in Fig. 6 at the inflow, all of the inviscid and viscous interfering bluntbody computations start with the appropriate freestream and oblique shock wave conditions as boundary conditions.

Viscous steady computations with or without impinging shock. To keep the study tractable, only two types of physical flow fields were chosen. The first is the viscous hypersonic blunt-body flow at $M_{\infty}=8.03$ and $T_{\infty}=122.1 \mathrm{~K}$ with a laminar Reynolds number of 387,750 based on the body diameter for ideal gases. The second problem (with the same flow conditions) is similar to the inviscid shock-onshock interaction where an oblique shock impinges on the bow shock of the blunt body at an angle of $19^{\circ}$ relative to the free stream. A more detailed flow field computation on the six types of shock patterns categorized by Edney [43] is presented in Ref. [21]. For the convergence study only one type of interaction, namely what is called the Type III interaction, is considered. Also the study is restricted to only one type of time stepping sequencing and only one value of the entropy correction parameter. The computational meshes (not shown) consist of 181 points in the circumferential direction and 91 points in the normal to the body direction and are highly clustered in the wall region to resolve the viscous layer.

At this point, it is important to point out that the time step sequence used for the viscous steady flows is very different from the inviscid study. Most of the inviscid computations use the same initial time step input together with a local time-stepping procedure throughout the entire iteration process. The time step sequence chosen for the viscous steady calculations is based on experience with a wide range of hypersonic flow simulations and consists of a sequence of fixed time steps with the procedure of doubling the time step every 100-400 iterations until the specified final time step is reached. The initial time step is $\Delta t=0.001$ which corresponds to a maximum Courant (CFL) number of 10 to 20 for the current problem and grid size. Larger values of the initial time step usually prevent convergence. The four specified time steps considered range from 0.001 to 0.008 with the corresponding CFL numbers ranging from 20 to 200. Much larger maximum CFL (or specified final time step) numbers are possible but do not improve the convergence rates. The value of the entropy correction parameter was fixed at $\tilde{\delta}=0.15$, again based on experience with a wide range of hypersonic flow field simulations.

The results of the blunt-body steady viscous flow obtained with the temporally second-order accurate algorithm (12) (hereafter referred to as the full matrix form) are shown in Fig. 8. Here algorithm (12) for the viscous computations means the appropriate three-point central differences of the viscous terms are added to the explicit and implicit operators of (12). Depicted are the Mach contours ranging from 0 to 8 in increments of 0.1 and the entropy contours normalized with the freestream value and ranging from 0 to 6.4 in increments of 0.1 . The final view in Fig. 8 is the $L_{2}$-norm residual history. The residual drops to machine accuracy $\left(10^{-14}\right)$ in less than 3200 steps. The corresponding results using the same algorithm (12a)-(12f) together with (13) (hereafter referred as the diagonal form) are illustrated in Fig. 9. No noticeable difference in the numerical results is observed in 

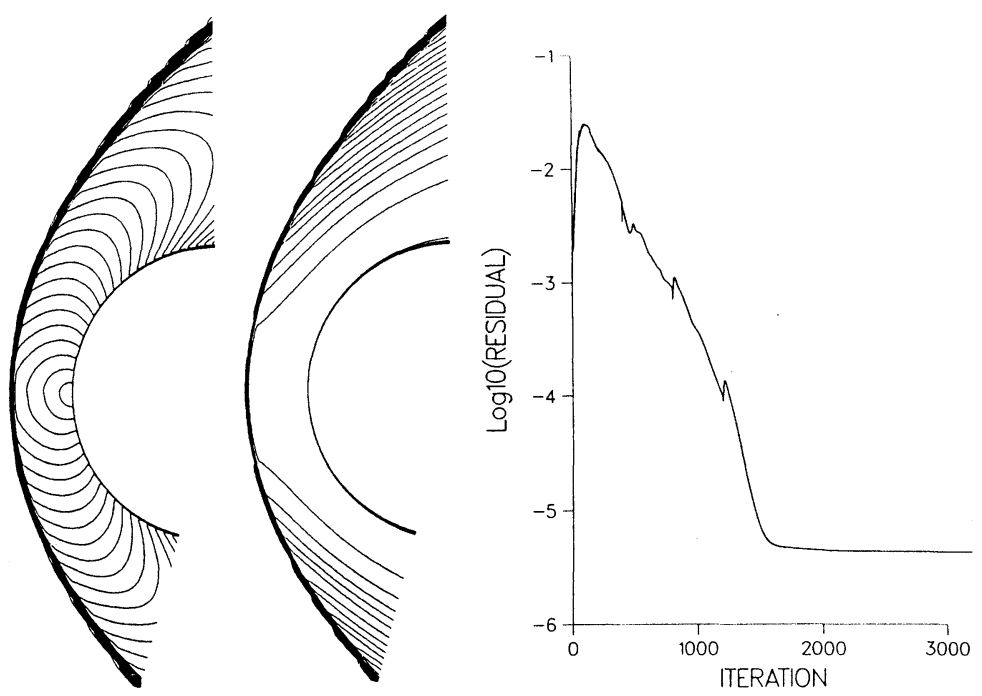

Fig. 9. Mach contours, entropy contours, and residual history for the steady viscous blunt-body flow computed by algorithm (12), (13) $\left(\theta=1, \omega=\frac{1}{2}\right.$, diagonal form) with $M_{\infty}=8.03, \operatorname{Re}_{D}=387,750$, $\gamma=1.4$, and laminar flow.

the Mach number or entropy contours. However, the residual curves are very different. The residual for the diagonal scheme reached a plateau of $5 \times 10^{-6}$ at 1500 steps and stayed at that level.

A more complex flow field which is far more difficult to compute is depicted in Figs. 10 and 11 . The results using the same second-order time accurate full matrix algorithm are shown in Fig. 10. The convergence rate is slower than for the bluntbody non-interfering case but is still satisfactory. The residual dropped seven orders of magnitude in 3000 steps. In both of the blunt-body flows with or without impinging shocks, steady state can be reached within 1000-1500 iterations. The extra iterations are needed only to bring the residual to a lower level but no change in the contour plots or surface pressures at least to within 3-4 digits of accuracy is observed. However, the results shown in Fig. 11 using the diagonal scheme are not satisfactory. The residuals dropped less than two orders of magnitude in 3000 steps. The noise appearing on the Mach number and entropy contours in the upper portion of the bow shock using the diagonal form of the scheme indicates that the algorithm has a problem reaching the converged steady-state solution.

All of the results obtained for Figs. 8-11 have the viscous terms included in the implicit operator. If the viscous terms are not included in the implicit operator, then the full matrix scheme becomes unstable for $\Delta t \geqslant 0.004$, whereas the diagonal scheme exhibits no change in convergence rate.

In summary, from the point of view based on the $L_{2}$-norm of the residuals, the best convergence rates were achieved by the full matrix form with the viscous terms included, since it allowed the residual to drop to machine accuracy $\left(10^{-14}\right)$. The 

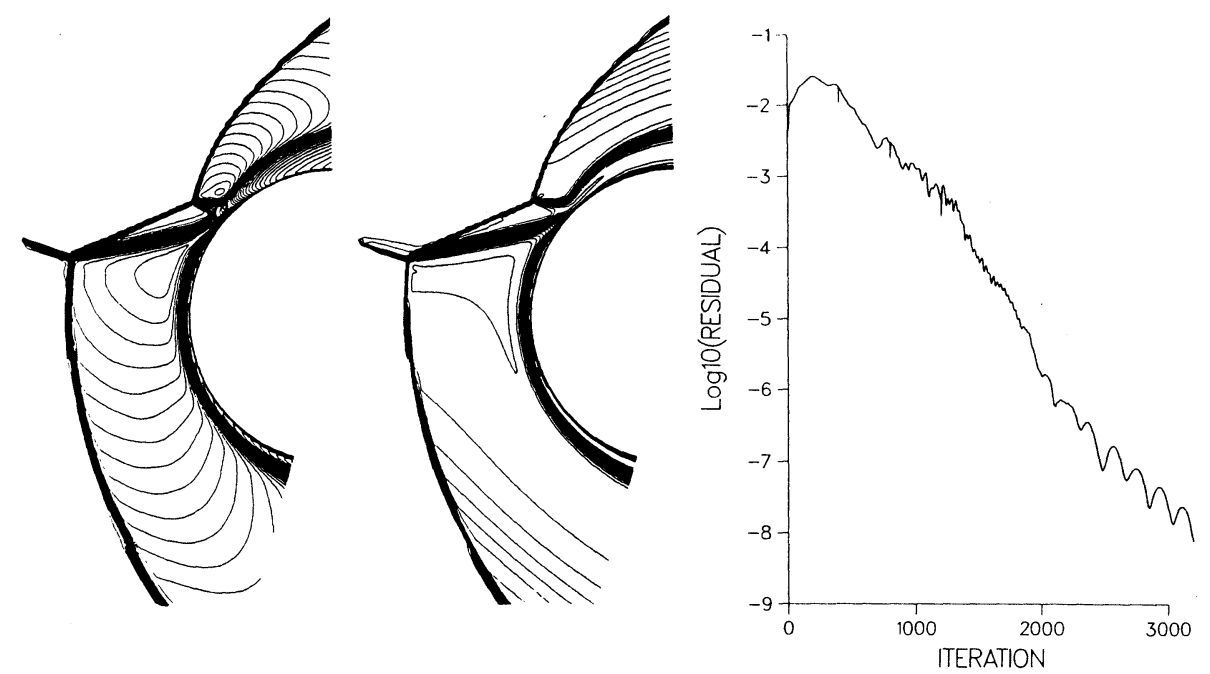

FIG. 10. Mach contours, entropy contours, and residual history for the steady viscous Type III shock interference flow computed by algorithm (12) $\left(\theta=1, \omega=\frac{1}{2}\right.$, full matrix form) with $M_{\infty}=8.03$, $\operatorname{Re}_{D}=387,750, \gamma=1.4$, and laminar flow.

diagonal form (13) did not fare too well. Although there is a substantial savings in operation count per iteration $(\sim 20 \%$ less $)$, the $L_{2}$-norm of the residual never dropped below $10^{-6}$ for all the time steps considered, and for the impinging shock case, the algorithm has a slight problem reaching the converged steady state. Moreover, the inclusion of the implicit viscous terms had little effect on the
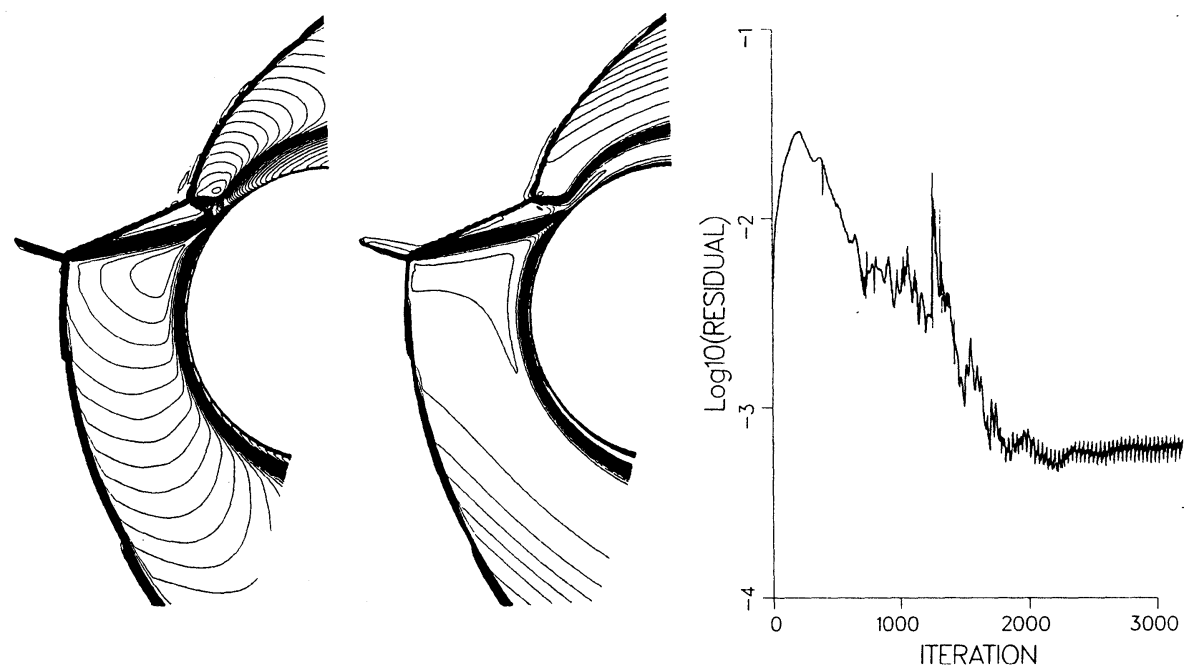

FIG. 11. Mach contours, entropy contours, and residual history for the steady viscous Type III shock interference flow computed by algorithm (12), (13) $\left(\theta=1, \omega=\frac{1}{2}\right.$, diagonal form) with $M_{\infty}=8.03$, $\operatorname{Re}_{D}=387,750, \gamma=1.4$, and laminar flow. 
diagonal form of the scheme but is important for the full matrix form of the scheme. One way of taking advantage of the low operations count ( $\sim 30 \%$ less $)$ of the diagonal form (without the implicit viscous terms) is to use the scheme as an efficient way of obtaining a rough solution (from freestream) for the initial input to the full matrix algorithm. The temporally second-order time-differencing scheme had a marginal but beneficial effect on the convergence rates when compared with the temporally first-order scheme.

Viscous steady and unsteady Mach 15 computations with impinging shock. Figure 12 illustrates the shock resolution of unsteady and steady thin-layer NavierStokes computations by the second-order time-accurate, full matrix algorithm (12). This steady test case is similar to the previous impinging shock study except the freestream Mach number is 15 , the impingement shock angle is $22.75^{\circ}$, the freestream temperature is $T_{\infty}=255.6 \mathrm{~K}$, and the Reynolds number based on the diameter is 186,000 . Shown are the Mach contours from 0 to 15 in increments of 0.1 . For the unsteady computation, the impingement shock at an angle of $22.75^{\circ}$ relative to the freestream moves downward across the bow-shock of the blunt body. The impingement shock velocity is $10 \%$ of the freestream velocity $\left(M_{\infty}=15\right)$. Although the impingement shock locations for the unsteady and steady computations are similar, the shock patterns are very different. A $241 \times 141$ non-adaptive grid is used for both computations. A time step of 0.002 (equivalent to a maximum CFL of 48) is used for steady-state computations whereas a time step of 0.0005 (equivalent to a maximum CFL of 10-12 at the vicinity of the boundary layer and a CFL of 1 at the rest of the flow field) is used for the unsteady computations. The steady-state solution can be reached in 1200 steps with a three order of magnitude
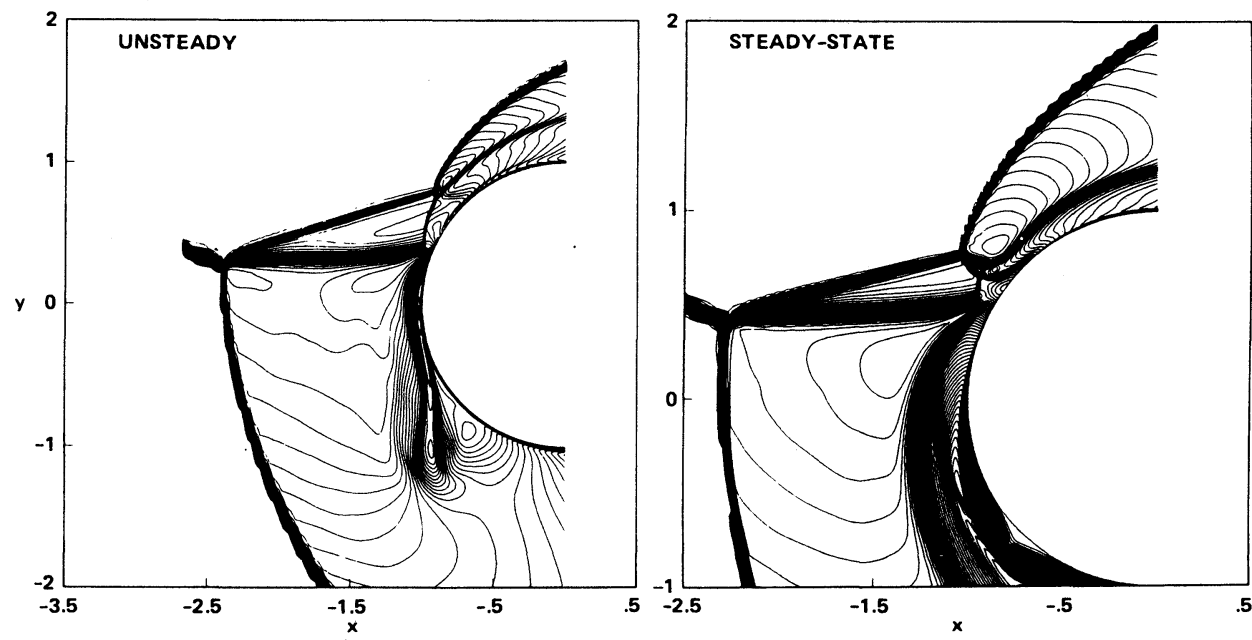

Fig. 12. The Mach contours of a two-dimensional viscous steady and unsteady hypersonic perfect gas computation by algorithm (12) $\left(\theta=1, \omega=\frac{1}{2}\right.$, full matrix form) with $M_{\infty}=15$ and $\operatorname{Re}_{D}=186,000$. 
drop in the $L_{2}$-norm residual. However, extra iterations are needed to bring the residual to a lower level but no change in the contour plots or surface pressures at least to within 3-4 digits of accuracy is observed. More detailed study of the surface pressure and heat transfer rate of these types of shock-on-shock steady and unsteady numerical simulations was reported in Refs. [21, 22].

\section{Concluding Remarks}

A two-parameter family of implicit time-accurate shock-capturing algorithms for hypersonic viscous flows has been developed. The proposed algorithms are formulated in finite volume and pseudo finite volume form and have been shown to be quite efficient and accurate for steady-state as well as unsteady viscous and inviscid hypersonic complex shock interactions. Some numerical aspects of these TVD-type algorithms that affect the convergence rate for hypersonic Mach numbers or real gas flows but have negligible effect on low Mach numbers or perfect gas flows are identified. Improvements have been made to the algorithms to speed up the convergence rate in the hypersonic flow regime. Even with the improvements, though, the convergence is, in general, slower for real gases than for a perfect gas. The nonsmoothness in the curve fits of Srinivasan et al. may be a major contributing factor in slowing down the convergence rate. Also, the convergence rate is, in general, slower for viscous flows than for inviscid steady flows.

\section{REFERENCES}

1. J.-L. Montagné, H. C. Yee, G. H. Klopfer, and M. VinokuR, in Proceedings, 2nd International Conference in Hyperbolic Problems, Aachen, West Germany, 1988, edited by J. Ballmann and R. Jeltsch (Vieweg, Braunschweig, 1989), p. 413; NASA TM-100074, 1988 (unpublished).

2. H. C. Yee And J. L. Shinn, in Proceedings, AIAA 8th Computational Fluid Dynamics Conference, Honolulu, Hawaii, 1987, AIAA-87-1116-CP, edited by W. J. McCroskey (AIAA, Washington, DC, 1987), p. 159.

3. R. J. LeVeque AND H. C. YeE, NASA TM-100075, March 1988 (unpublished).

4. H. C. YeE, Comput. Math. Appl. A 12, 413 (1986).

5. H. C. Yee AND A. Harten, AIAA J. 25, 266 (1987).

6. H. C. YeE, J. Comput. Phys. 68, 151 (1987).

7. B. van Leer, J. Comput. Phys. 32, 101 (1979).

8. K. W. ANderson, J. L. Thomas, and B. van LeER, AiAA J. 24, 1453 (1986).

9. S. Osher and S. ChaKravarthy, The IMA Volumes in Mathematics and Its Applications 2, Minneapolis, MN, 1985, edited by C. Dafermos et al. (Springer-Verlag, New York, 1986), p. 229.

10. P. Woodward and P. Colella, I. Comput. Phys. 54, 115 (1984).

11. J.-L. Montagne, H. C. Yee, AND M. VinokuR, in Proceedings, 7th GAMM Conference on Numerical Methods in Fluid Mechanics, Belgium, 1987; NASA TM-86839, July 1987 (unpublished).

12. H. C. YeE, NASA TM-89464, May 1987 (unpublished).

13. P. L. RoE, J. Comput. Phys. 43, 347 (1981).

14. M. VINOKUR, NASA Contractor Report 177512, Dec. 1988 (unpublished).

15. M. VINOKUR AND Y. LiU, AIAA-88-0127, Reno, Nevada, January 1988 (unpublished). 
16. J. L. Steger and R. F. Warming, J. Comput. Phys. 40, 263 (1981).

17. B. vAN LEER, ICASE Report No. 82-30, Sept. 1982 (unpublished).

18. M. VinOKUR AND J.-L. MontagnE, NASA Contractor Report 177513, Dec. 1988 (unpublished).

19. A. Harten, SIAM J. Num. Anal. 21, 1 (1984).

20. Y. Takakura, T. Ishiguro, and S. Ogawa, in Proceedings, AIAA 8th Computational Fluid Dynamics Conference, Honolulu, Hawaii, 1987, AIAA-87-1151-CP, edited by W. J. McCroskey (AIAA, Washington, DC, 1987), p. 537.

21. G. Klopfer and H. C. Yee, "Viscous Hypersonic Shock on Shock Interaction on Blunt Cowl Lips," AIAA-88-0233, AIAA 26th Aerospace Sciences Meeting, Reno, Nevada, Jan. 11-14, 1988 (unpublished).

22. G. KloPfer, H. C. Yee, AND P. KutleR, in Proceedings, 11th International Conference on Numerical Methods in Fluid Dynamics, Williamsburg, Virginia, 1988, edited by D. Dwoyer (Springer-Verlag, New York, 1989), p. 234; NASA TM-100096, April 1988 (unpublished).

23. P. L. RoE, Lectures in Applied Mathematics, Vol. 22, edited by Osher et al. (Amer. Math. Soc., Providence, RI, 1985), p. 163.

24. P. L. RoE, ICASE Report No. 84-53, Oct. 1984 (unpublished).

25. S. F. DAvis, ICASE Report No. 84-20, June 1984 (unpublished).

26. H. C. YeE, NASA TM-86839, Sept. 1985 (unpublished).

27. T. J. BARTH, AIAA-87-0595, Reno, Nevada, 1987 (unpublished).

28. M. VINOKUR, NASA Contractor Report 177416, June 1986 (unpublished).

29. H. C. YeE, in Proceedings, 10th Int. Conf. on Numerical Methods in Fluid Dynamics, Beijing, China, 1986, edited by F. G. Zhuang and Y. L. Zhu (Springer-Verlag, Berlin, 1986), p. 677.

30. T. H. Pulliam and J. L. Steger, AIAA Paper 85-0360, Reno, Nevada, 1985 (unpublished).

31. H. C. YEE, NASA TM-81265, 1981 (unpublished).

32. A. Jameson, W. Schmidt, and E. Turkel, AIAA Paper 81-1259, Palo Alto, CA, 1981 (unpublished).

33. A. Harten and J. M. Hyman, J. Comput. Phys. 50, 235 (1983).

34. J. C. TANNEhill, T. L. Holst, and J. V. Rakich, AIAA J. 14, 204 (1976).

35. P. A. Gnoffo and R. S. MCCandless, AIAA Paper 86-0230, Reno Nevada, Jan. 1986 (unpublished).

36. P. L. Roe AND J. Pike, Computing Methods in Applied Sciences and Engineering, Paris, France, 1983, edited by R. Glowinski and J.-L. Lions (North-Holland, Amsterdam, 1984), p. 499.

37. H. C. Yee, R. F. Warming, and A. Harten, J. Comput. Phys. 57, 327 (1985).

38. H. C. Yee AND P. Kutler, NASA TM-85845, August 1983 (unpublished).

39. S. SRInivasan, J. C. Tannehill, ANd K. J. Weilmunster, ISU-ERI-Ames 86401; ERI Project 1626; CFD15 (unpublished).

40. J. C. Tannehill, T. J. Holst, J. V. Rakich, and J. W. Keyes, aiaA J. 14, 539 (1976).

41. J. A. White and C. M. RhIE, AIAA-87-1895, San Diego, CA, June 1987 (unpublished).

42. K. Morgan, J. Peraire, R. R. Thareja, and J. R. Stewart, in Proceedings, AiaA 8th Computational Fluid Dynamics Conference, Honolulu, Hawaii, 1987, edited by W. J. McCroskey (AIAA, Washington, DC, 1987), p. 749.

43. B. E. EDNEY, FFA Report 115, February 1968, The Aeronautical Research Institute of Sweden, Stockholm, Sweden (unpublished). 